ARTICLE

DOI: $10.1038 / s 41467-018-06819-5$

\title{
Decoding the intensity of sensory input by two glutamate receptors in one $C$. elegans interneuron
}

\author{
Wenjuan Zou ${ }^{1,2,3}$, Jiajun $\mathrm{Fu}^{1,4}$, Haining Zhang ${ }^{1,2}$, Kang Du², Wenming Huang ${ }^{2}$, Junwei Yu', Shitian $\mathrm{Li}^{3}$,

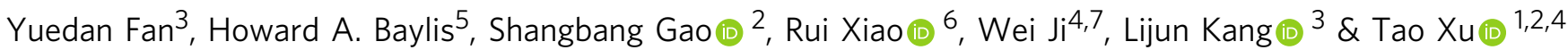

How neurons are capable of decoding stimulus intensity and translate this information into complex behavioral outputs is poorly defined. Here, we demonstrate that the $C$. elegans interneuron AIB regulates two types of behaviors: reversal initiation and feeding suppression in response to different concentrations of quinine. Low concentrations of quinine are decoded in AIB by a low-threshold, fast-inactivation glutamate receptor GLR-1 and translated into reversal initiation. In contrast, high concentrations of quinine are decoded by a highthreshold, slow-inactivation glutamate receptor GLR-5 in AIB. After activation, GLR-5 evokes sustained $\mathrm{Ca}^{2+}$ release from the inositol 1,4,5-trisphosphate $\left(\mathrm{IP}_{3}\right)$-sensitive $\mathrm{Ca}^{2+}$ stores and triggers neuropeptide secretion, which in turn activates the downstream neuron RIM and inhibits feeding. Our results reveal that distinct signal patterns in a single interneuron AIB can encode differential behavioral outputs depending on the stimulus intensity, thus highlighting the importance of functional mapping of information propagation at the single-neuron level during connectome construction.

\footnotetext{
${ }^{1}$ National Laboratory of Biomacromolecules, CAS Center for Excellence in Biomacromolecules, Institute of Biophysics, Chinese Academy of Sciences, Beijing 100101, China. ${ }^{2}$ Key Laboratory of Molecular Biophysics of the Ministry of Education, College of Life Science and Technology, Huazhong University of Science and Technology, Wuhan 430074, China. ${ }^{3}$ Institute of Neuroscience and Department of Neurosurgery of the First Affiliated Hospital, Department of Neurobiology, NHC and CAMS Key Laboratory of Medical Neurobiology, Zhejiang University School of Medicine, Hangzhou 310058, China. ${ }^{4}$ College of Life Sciences, University of Chinese Academy of Sciences, Beijing 100049, China. ${ }^{5}$ Department of Zoology, University of Cambridge, Cambridge CB2 3EJ, UK. ${ }^{6}$ Department of Aging and Geriatric Research, Institute of Aging, College of Medicine, Center for Smell and Taste, University of Florida, Gainesville 32610 FL, USA. ${ }^{7}$ Center for Biological Instrument Development, Core Facility for Protein Research, Institute of Biophysics, Chinese Academy of Sciences, Beijing 100101, China. These authors contributed equally: Wenjuan Zou, Jiajun Fu, Haining Zhang. Correspondence and requests for materials should be addressed to W.J. (email: jiwei@moon.ibp.ac.cn) or to L.K. (email: kanglijun@zju.edu.cn) or to T.X. (email: xutao@ibp.ac.cn)
} 
D ecoding stimulus intensity is a fundamental property of sensory systems. Organisms use this information to adapt their behavior in response to stimulus strength. For instance, in many vertebrates and invertebrates salt can be either attractive or repulsive depending on its concentration ${ }^{1-3}$. This leads to important questions in neuroscience, how is the stimulus intensity encoded at different stages of neural processing and how is this information translated into complex behavioral outputs?

Simple organisms such as C. elegans provide premier platforms to tease out how signaling molecules and neuronal circuits generate complex behaviors. C. elegans is equipped with a small nervous system, merely 302 neurons and 7000 synapses, of which the complete connectome is known ${ }^{4}$. Moreover, C. elegans responds to complex environmental cues associated with the senses of smell, taste, touch, and temperature, all of which evoke multifaceted behavioral responses such as locomotion, feeding, dauer formation, social behavior, and also learning and memory $^{5-9}$. Like many vertebrates and invertebrates, C. elegans is capable of decoding stimulus intensity and translating it into multifaceted behavioral outputs. For example, anterior gentle touch typically triggers short reversals followed by forward movement without a direction change. By contrast, anterior harsh touch evokes long reversals, which are often followed by a directional change through omega turns ${ }^{10}$.

Previously we and others have shown that quinine, a type of bitter alkaloid and repellent to C. elegans, can generate two types of behavior: reversal initiation and feeding suppression ${ }^{11,12}$. This phenomenon provokes some intriguing questions. First, do reversal initiation and feeding suppression always occur simultaneously when encountering quinine or do they occur differentially depending on the stimulus intensity? Additionally, what are the underlying neural circuitry and molecular mechanisms for signal decoding, processing, and different type of behavioral output?

Several key components in the neural circuit that control $C$. elegans reversal initiation have been previously identified ${ }^{13-17}$. In particular, ASH is the main sensory neuron responsible for the detection of repellents detection including quinine while ASK plays a minor role ${ }^{11,18}$. Moreover, a group of command interneurons (AVA, AVD, and AVE) play an important role in the initiation of reversals. The ablation of AVA and AVD severely reduces the rate of spontaneous reversals but does not abolish them entirely ${ }^{15,19}$. ASH sends synapses to both AVA and $\mathrm{AIB}^{4}$. Glutamate released from the ASH sensory neuron is necessary to activate both AIB and AVA in reversal initiation ${ }^{15,16}$. Mutants in a glutamate receptor $g l r-1$ are significantly defective in the reversal response ${ }^{15-17}$. Targeted expression of GLR-1 to the interneurons AVA or AIB results in a partial rescue of the reversal response, supporting a model in which the AIB and AVA pathways function in parallel, but not completely redundantly ${ }^{15}$. It has also been suggested that RIM acts downstream of $\mathrm{AIB}$ and is involved in reversal behavior ${ }^{13,15}$. However, RIM also sends chemical and electrical synapses to AIB and generates variability in odor-induced reversals ${ }^{20}$.

One of the most prominent behaviors in animals is feeding. In contrast to locomotion, the circuitry of feeding regulation in $C$. elegans has not been extensively characterized. Previously we have identified a central flip-flop circuit that integrates two contradictory sensory inputs in regulating feeding behavior ${ }^{12}$. The central integration circuit receives inputs from different sensory modalities in such a way that neurons sensing attractive inputs (AWA and AWC neurons) are linked to NSM neurons to facilitate pharyngeal pumping and neurons sensing repellents (ASH neuron) transmit signals to RIM/RIC neurons to suppress pumping. Sensation of quinine suppresses feeding through ASH-RIM pathway. Therefore, it appears that the signals encoding the reversal initiation and feeding suppression converge on RIM. The question remains as to how the same sensory signal is decoded and transmitted to the same interneurons to control two types of behavioral outputs.

By integrating $\mathrm{Ca}^{2+}$ imaging, optogenetics, genetic manipulation, and electrophysiology at the single-neuron resolution, here we reveal that a single-interneuron type, AIB, decodes the intensity of quinine stimuli and encodes two types of behavioral outputs, reversal initiation and feeding suppression. In AIB neuron, low-concentration quinine input is decoded by a lowactivation threshold, fast-inactivation glutamate receptor GLR-1 to mediate the reversal response, whereas high-concentration quinine stimulation is decoded by a high-activation threshold, slow-inactivation glutamate receptor GLR-5 to mediate feeding suppression. These two behavioral outputs are encoded in AIB by distinct $\mathrm{Ca}^{2+}$ response patterns: small, transient $\left[\mathrm{Ca}^{2+}\right]_{\mathrm{i}}$ spikes for short reversals, and large, sustained $\mathrm{Ca}^{2+}$-release from $\mathrm{IP}_{3}$ sensitive $\mathrm{Ca}^{2+}$ store for long reversals and feeding suppression. Together, our data reveal the circuit, synaptic, molecular, and intracellular mechanisms by which $C$. elegans decodes the intensity of quinine stimulus and encodes two behavioral outputs.

\section{Results}

Simultaneous $\mathrm{Ca}^{2+}$ imaging and behavioral tracking. To further dissect the circuit connections and molecular mechanisms that regulate both feeding and locomotion, we developed a fast tracking system called iCaN (imaging the Calcium activity of Nematodes) that not only analyzes locomotion and feeding behavior, but also simultaneously monitors intracellular $\mathrm{Ca}^{2+}$ concentrations $\left(\left[\mathrm{Ca}^{2+}\right]_{\mathrm{i}}\right)$ in the neurons of freely moving worms. Briefly, iCaN is equipped with a dual objective system: (1) a lowmagnification objective above the sample to track the movement of free-moving worms and to record feeding behavior (quantified by pharyngeal pumping rate); (2) a high-magnification objective below the sample to monitor neuronal $\left[\mathrm{Ca}^{2+}\right]_{\mathrm{i}}$ activity (Supplementary Figure 1). The recorded locomotion is fed back to an $\mathrm{x}-\mathrm{y}$ stage to center the worm in the field-of-view of both objectives. This design ensures fast 4D imaging of worm neurons at high resolution.

Employing the iCaN system, we confirmed that repellents, such as quinine, could generate two kinds of behavioral outputs, reversals in locomotion and suppression of feeding. Interestingly, the two behavioral outputs were not always induced simultaneously but rather depended on the concentration of quinine. Low concentrations $(1 \mathrm{mM})$ initiated frequent reversals but did not significantly suppress pumping rate (Fig. 1a-c), whereas high concentrations $(4-5 \mathrm{mM})$ induced long-lasting reversals and pumping suppression (Fig. 1d, e).

In C. elegans, $\mathrm{ASH}$ is the main sensory neuron responsible for quinine detection and feeding inhibition ${ }^{11,12}$. AIB is the first layer interneuron downstream of ASH that is involved in reversal initiation ${ }^{4,15,16}$. Simultaneous monitoring of behavior and $\left[\mathrm{Ca}^{2+}\right]_{\mathrm{i}}$ in the AIB neurons revealed that low concentrations of quinine-induced small, transient $\left[\mathrm{Ca}^{2+}\right]_{\mathrm{i}}$ increases (Fig. 1a, c), while high concentrations of quinine-induced large, sustained $\left[\mathrm{Ca}^{2+}\right]_{\mathrm{i}}$ elevations (Fig. 1d, e). We found a good correlation between AIB $\left[\mathrm{Ca}^{2+}\right]_{\mathrm{i}}$ activities and reversal behavior. Upon weak stimulation ( $1 \mathrm{mM}$ quinine), $\mathrm{AIB}\left[\mathrm{Ca}^{2+}\right]_{\mathrm{i}}$ transients peaked at the end of a reversal and gradually returned to baseline with the resumption of forward movement (Fig. 1f), which are consistent with previous studies ${ }^{15,21}$. In the presence of higher concentrations of quinine, AIB $\left[\mathrm{Ca}^{2+}\right]_{\mathrm{i}}$ activity was sustained throughout the long-lasting reversal and began to decline at the resumption of forward movement (Fig. 1g). On the other hand, while the low concentrations of quinine-induced transient AIB activities 
a

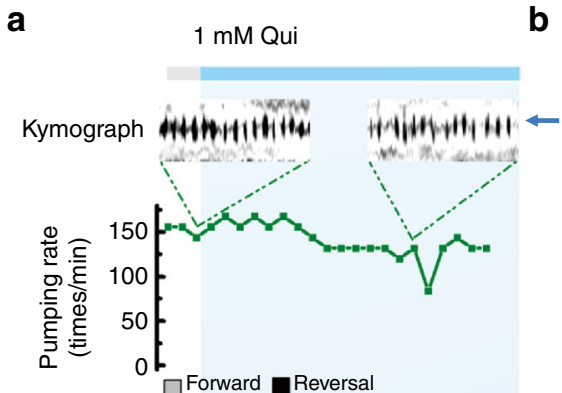

Locomotion $\square$ Forward Reversal U \}

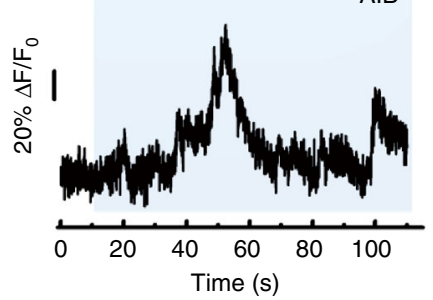

C

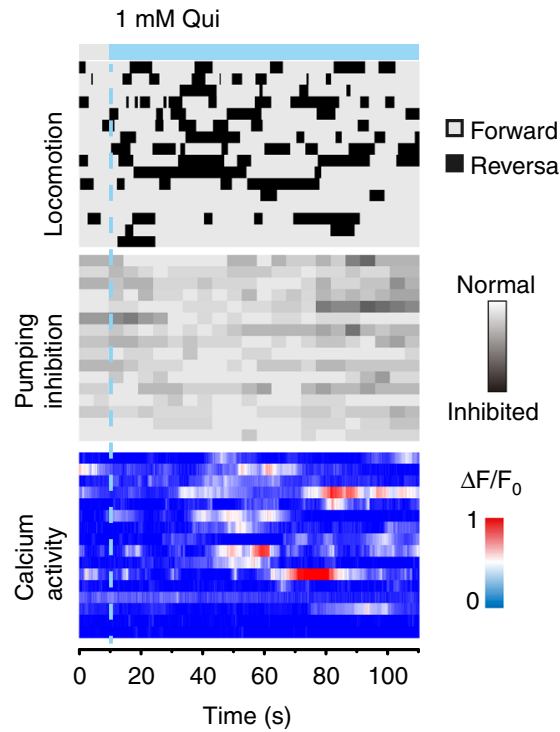

b

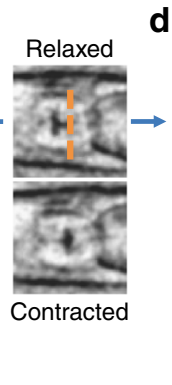

d
$4 \mathrm{mM}$ Qui
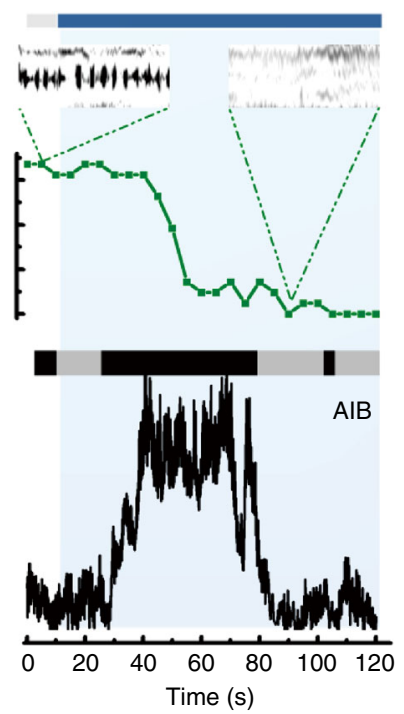

e

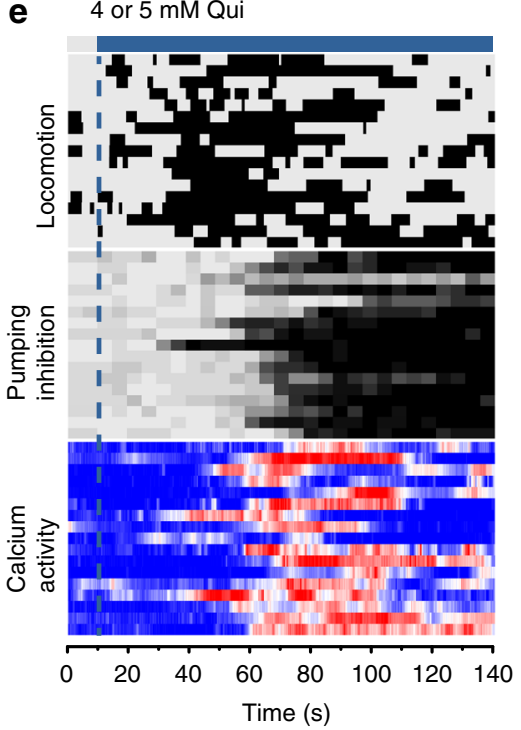

f

$1 \mathrm{mM}$ Qui

AIB::G-CaMP

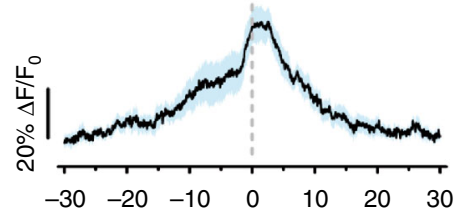

g 4 or $5 \mathrm{mM}$ Qui AIB::G-CaMP

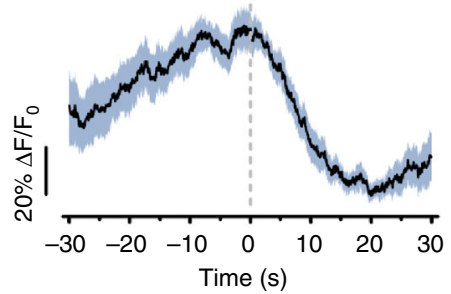

Reversal ends at $t=0$

h
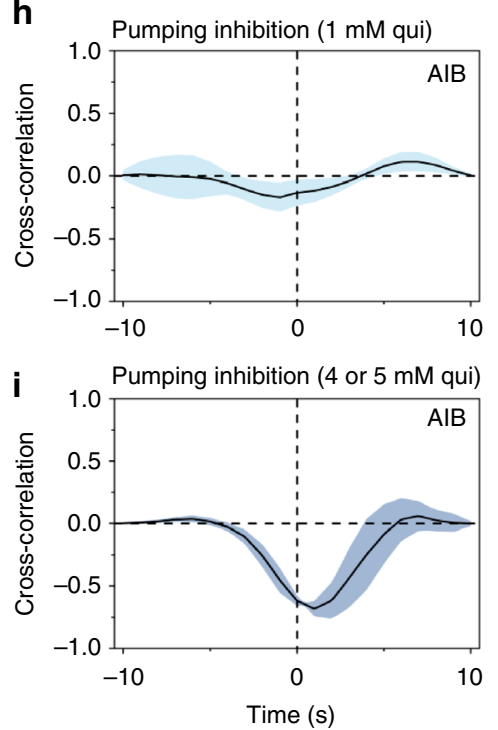

Fig. 1 Different strengths of quinine elicit different behavioral outputs: reversal initiation and feeding inhibition. $\mathbf{a}, \mathbf{b}, \mathbf{d} 1 \mathrm{mM}$ quinine initiated frequent reversal, induced small and transient $\left[\mathrm{Ca}^{2+}\right]_{\mathrm{i}}$ activities in AIB, but did not significantly suppress pumping rate. In contrast, $5 \mathrm{mM}$ quinine-induced pumping suppression, reversal initiation and large, sustained $\left[\mathrm{Ca}^{2+}\right]_{i}$ elevations in AIB. Pumping rate and locomotion were assayed with the iCaN system in a freely behaving worm, while intracellular calcium transients from AIB were simultaneously imaged. The kymographs denote the pumping pulse of feeding in $5 \mathrm{~s}$ selected before and after the application of quinine. The grinder muscle moving from relaxed position to contracted position (b) and returning to relaxed position represents a pump, which is shown as a spike in the kymographs (a, d). Orange dash line (b) indicates the position selected for the kymographs. The ethograms describing the locomotion behavior were generated automatically from the worm's posture and the direction of locomotion acquired by the iCaN system. c, e Locomotion, pumping behavior, and AIB calcium dynamics in response to low (c) and high (e) concentration of quinine in freely behaving worms. Traces are ordered according to the time of quinine application (dash lines). $n \geq 16$ worms. $\mathbf{f}, \mathbf{g}$ AlB calcium level exhibited an increase during reversals and a decrease at the end of reversals induced by both low (f) and high concentration ( $\mathbf{g}$ ) of quinine. $n=9$ worms for high concentration and $n=$ 12 worms for low concentration. $\mathbf{h}$, i Cross-correlation analysis exhibited that AIB calcium activity correlates with pumping inhibition at high concentrations of quinine condition but not at low concentration. Shading around traces indicates error bars (s.e.m.). $n \geq 15$ worms

showed little correlation with pumping suppression (Fig. 1h), the high concentrations of quinine-triggered strong, sustained AIB activities correlated well with pumping suppression (Fig. 1i). Collectively, these results confirm that AIB is involved in locomotion reversal ${ }^{15,21}$ and, more importantly, suggested a previously unknown function of AIB in regulating feeding behavior.
AIB regulates reversal initiation and feeding suppression. To further confirm the functional specificity of AIB neurons in transducing quinine sensory inputs to feeding inhibition, we kill AIB and other first layer interneurons (AIA, AIZ, and AIY) individually using both laser ablation and neuron-specific expression of apoptosis factor CED-3 (Fig. 2a). Both laser and chemical ablation of AIB, but not other interneurons, abolished 
a

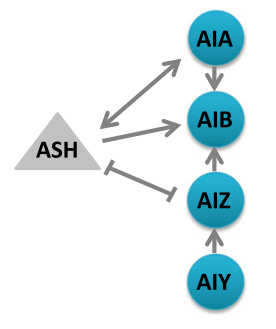

b

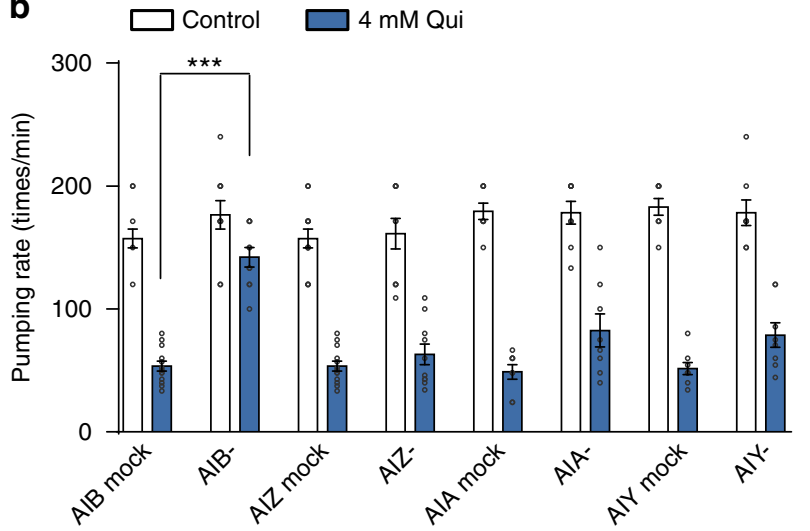

C

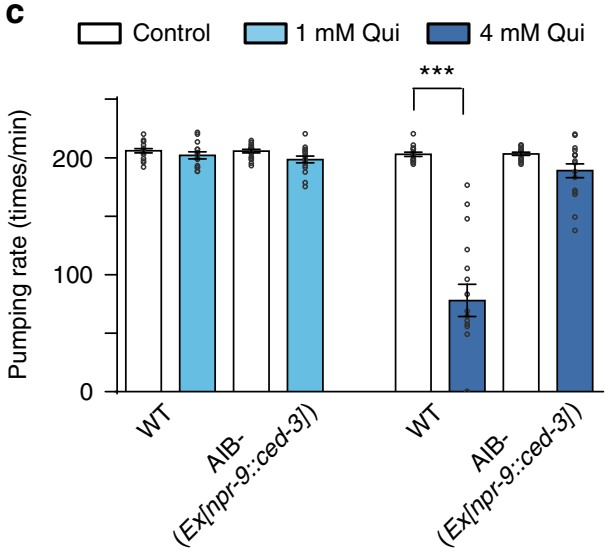

d

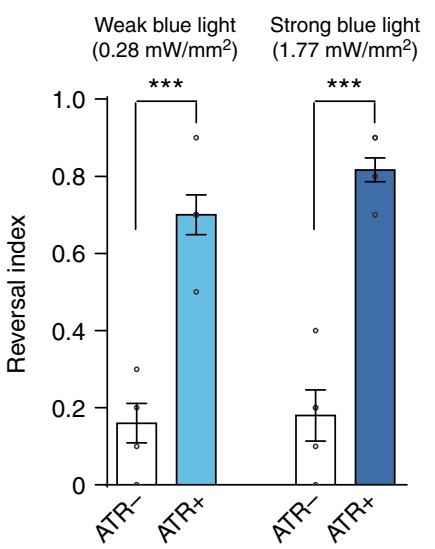

e

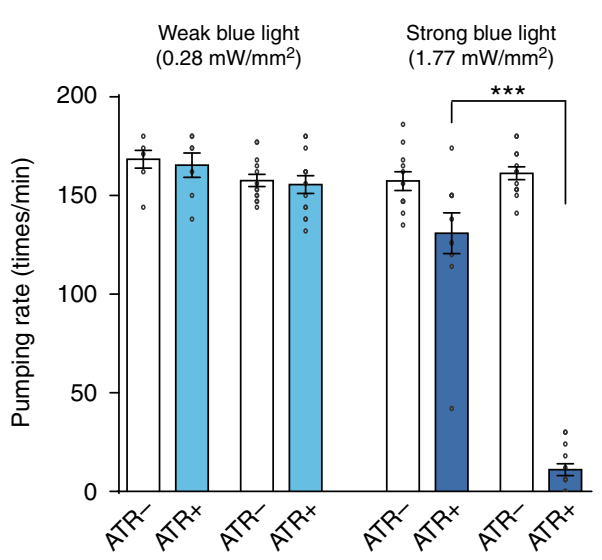

f

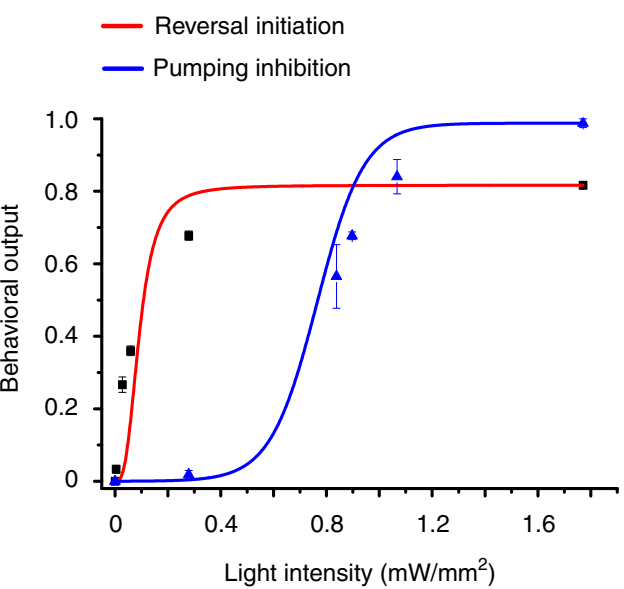

Fig. $2 \mathrm{AIB}$ is required for both reversal initiation and pumping inhibition. a Schematic showing the connectivity of ASH and the first layer interneurons (AIA, $\mathrm{AIB}, \mathrm{AIZ}$, and $\mathrm{AIY}$ ). b, $\mathbf{c}$ Laser ablation (b) and chemical deletion (c) of AIB exhibited a significantly defect in high-concentration quinine-induced pumping inhibition. For chemical deletion of AIB, CED-3 was transgenetically expressed in AIB driven by the $n p r-9$ promoter. Error bars: s.e.m.. Laser ablation: $n \geq 8$ worms; Chemical deletion: $n=16$ worms. ${ }^{\star \star *} p<0.001$ ( $t$ test). d, e Optogenetic stimulation of AIB evoked by either low power $\left(0.28 \mathrm{~mW} \mathrm{~mm}{ }^{-2}\right.$ ) or high power $\left(1.77 \mathrm{~mW} \mathrm{~mm}^{-2}\right)$ blue light $(470 \mathrm{~nm} ; 30 \mathrm{~s}$ pulse) triggered reversals, but only by high power blue light-induced pumping inhibition. Worms expressing ChR2 specifically in AIB under the npr-9 promoter were tested. The transgenic animals cultured on ATR-free plates were taken as control. d Reversal behavior. e Pumping behavior. Error bars: s.e.m.. Reversal index, $n \geq 5$ groups, and 10 worms/group; Pumping rate, $n \geq 10$ worms. ${ }^{\star \star \star} p<0.001$ ( $t$ - test). $\mathbf{f}$ Reversal initiation and pumping inhibition triggered by optogenetic activation of AIB. Worms expressed ChR2 in AIB were challenged with varying intensities of blue light to tune the activity of AIB. Reversal initiation and pumping inhibition were plotted as a function of blue light intensities. Lower light intensities were required for triggering reversals. Error bars: s.e.m. $n \geq 10$ worms

quinine-induced feeding inhibition (Fig. 2b, c). Chemical ablation of AIB also blocked low-concentration quinine-induced reversal initiation (Supplementary Figure 2), consistent with its reported role in regulating reversal locomotion ${ }^{15,21}$. Interestingly, highconcentration quinine-induced reversals were only partially blocked by AIB ablation, suggesting a redundant circuit independent of AIB in initiating reversal locomotion.

If AIB indeed regulates both reversal initiation and feeding suppression as suggested by our neuron ablation experiments, acute stimulation of AIB should affect both behavioral outputs. To test this, we took an optogenetic approach by expressing the light-gated cation channel channel rhodopsin-2 (ChR2) specifically in AIB using the $n p r-9$ promoter ${ }^{15,22}$. As predicted, we found that the behavioral outputs stimulated by AIB activation were light strength-dependent. Namely, weak activation of AIB by low intensity $\left(0.28 \mathrm{~mW} \mathrm{~mm}^{-2}\right)$ laser illumination $(488 \mathrm{~nm})$ elicited reversal but not feeding suppression, whereas sixfold stronger laser illumination $\left(1.77 \mathrm{~mW} \mathrm{~mm}^{-2}\right)$ elicited both reversal and feeding suppression (Fig. 2d, e). Plotting behavioral outputs against stimulation light intensities clearly suggested distinct sensitivities of two different behaviors to AIB photoactivation (Fig. 2f), which further supports that higher intensity of sensory inputs on AIB elicits additional behavioral outputs (Fig.1). Taken together, the results of $\left[\mathrm{Ca}^{2+}\right]_{\mathrm{i}}$ imaging under native condition, neuron ablation and optogenetic experiments all strongly suggest that AIB acts downstream of ASH to mediate two quinine-induced behavioral outputs in a stimulus intensitydependent manner: reversal initiation and feeding suppression. As locomotion and feeding are two types of behavioral outputs, we next sought to determine how different intensities of a single sensory input are decoded in AIB to produce this bifurcating pattern of commands to two behaviors.

Glutamate induces reversal initiation and feeding suppression. It has been reported that glutamate released from ASH is involved in nose touch-evoked reversal response ${ }^{14,15}$. Thus, we examined whether quinine-induced reversal locomotion and feeding suppression both require glutamate signaling from ASH to AIB. eat-4 encodes a vesicular glutamate transporter, ablation of which 
causes deficiency in glutamatergic transmission ${ }^{23,24}$. We observed that both reversal initiation and feeding suppression induced by quinine were abolished in the eat-4 mutants and were drastically reduced by ASH-specific RNAi of eat-4 (Fig. 3). Neuron-specific expression of EAT-4 in ASH neurons partially rescued reversal initiation in response to low quinine concentrations and restored reversal initiation (Fig. 3a) and feeding suppression (Fig. 3b) in response to high concentrations of quinine. Furthermore, highconcentration quinine-induced $\left[\mathrm{Ca}^{2+}\right]_{\mathrm{i}}$ responses in AIB were substantially reduced in the eat- 4 mutants (Fig. 3c, Supplementary Figure 3). Taken together, these results suggest that both low and high-concentration quinine-induced behavioral outputs are dependent on glutamate signaling from ASH.

GLR-1 and GLR-5 decode the intensity of quinine inputs. Next, we set to address how glutamate released from ASH could activate AIB. Three excitatory glutamate receptors, GLR-1, GLR-2, and GLR-5, and an inhibitory glutamate receptor AVR-14 have been shown to be expressed in $\mathrm{AIB}^{23,25}$. Our behavioral experiments showed that both low and high concentrations of quinine could initiate reversal in wild-type worms, $g l r-2, g l r-5$, and avr-14 mutants, but not in $g l r-1$ mutant worms (Fig. 4a, Supplementary Figure 4). Moreover, AIB-specific rescue of GLR-1 significantly restored the reversal response to quinine in the glr-1 mutant worms, confirming the action site of GLR-1 in AIB. On the other hand, glr-5 mutants, but not other glutamate receptor mutants, showed defects in feeding suppression induced by high-quinine concentrations (Fig. 4b, Supplementary Figure 4). This defect could be largely rescued by restoring expression of GLR- 5 in the AIB neuron of $g l r-5$ mutant background. Furthermore, $g l r-1 ; g l r-5$ double mutant and $g l r-1 ; g l r-5$;avr-14 triple mutant exhibited similar defects as $g l r-1$ and $g l r-5$ single mutants in reversal initiation and feeding suppression, respectively (Fig. 4a, b, Supplementary Figure 4). These results suggest that in response to ASH sensory inputs, GLR-1 and GLR-5, but not AVR-14, act independently in AIB neurons to mediate reversal initiation and feeding suppression, respectively.

To collect more evidence, we again employed optogenetic approach to stimulate ASH and record behavioral outputs under different strengths of stimulation and in different mutant background. We noticed that about 10 fold less power $(0.031$ $\mathrm{mW} \mathrm{mm}{ }^{-2}$ ) was required to excite ASH and initiate reversal (Fig. 4c) than that for AIB (Fig. 2). Importantly, reversal initiation was largely abolished in $g l r-1$ but not in $g l r-5$ mutants under both weak (Fig. 4c) and strong (Fig. 4d) ASH stimulation. In contrast, pumping suppression only occurred with strong ASH stimulation and was persisted in $g l r-1$ mutant but was significantly decreased in $g l r-5$ mutant (Fig. $4 \mathrm{~d}$ ).

To further illustrate the role of GLR-1 and GLR-5 in controlling AIB activity, we recorded quinine-induced $\left[\mathrm{Ca}^{2+}\right]_{\mathrm{i}}$ responses in AIB in the absence of either gene. As shown in Fig. 4e, f, low-concentration $(1 \mathrm{mM})$ quinine-induced $\left[\mathrm{Ca}^{2+}\right]_{\mathrm{i}}$ elevations in AIB were largely abolished in the absence of GLR-1 but not GLR-5. At high quinine concentration $(5 \mathrm{mM})$, whereas the peak amplitudes of $\left[\mathrm{Ca}^{2+}\right]_{\mathrm{i}}$ were comparable, the $\left[\mathrm{Ca}^{2+}\right]_{\mathrm{i}}$ elevations were sustained in $g l r-1$ but became transient in $g l r-5$ mutants (Fig. 4g, h). Analysis of the full width at half maximum (FWHM) of $\left[\mathrm{Ca}^{2+}\right]_{\mathrm{i}}$ elevations demonstrated longer FWHMs in $g l r-1$ than those in $g l r-5$ mutants (Fig. $4 \mathrm{~h}$ ). The $\left[\mathrm{Ca}^{2+}\right]_{\mathrm{i}}$ elevations were the longest in wild-type AIB neurons, probably due to an additive contribution of GLR-1 and GLR-5 to $\left[\mathrm{Ca}^{2+}\right]_{i}$ signals. Furthermore, high-concentration $(5 \mathrm{mM})$ quinine-induced $\left[\mathrm{Ca}^{2+}\right]_{\mathrm{i}}$ elevations in AIB were largely reduced in $g l r-1 ; g l r-5$; avr14 triple mutant, but not in glr-1;avr-14 double mutant (Supplementary Figure 4c), suggesting that AVR-14 is not
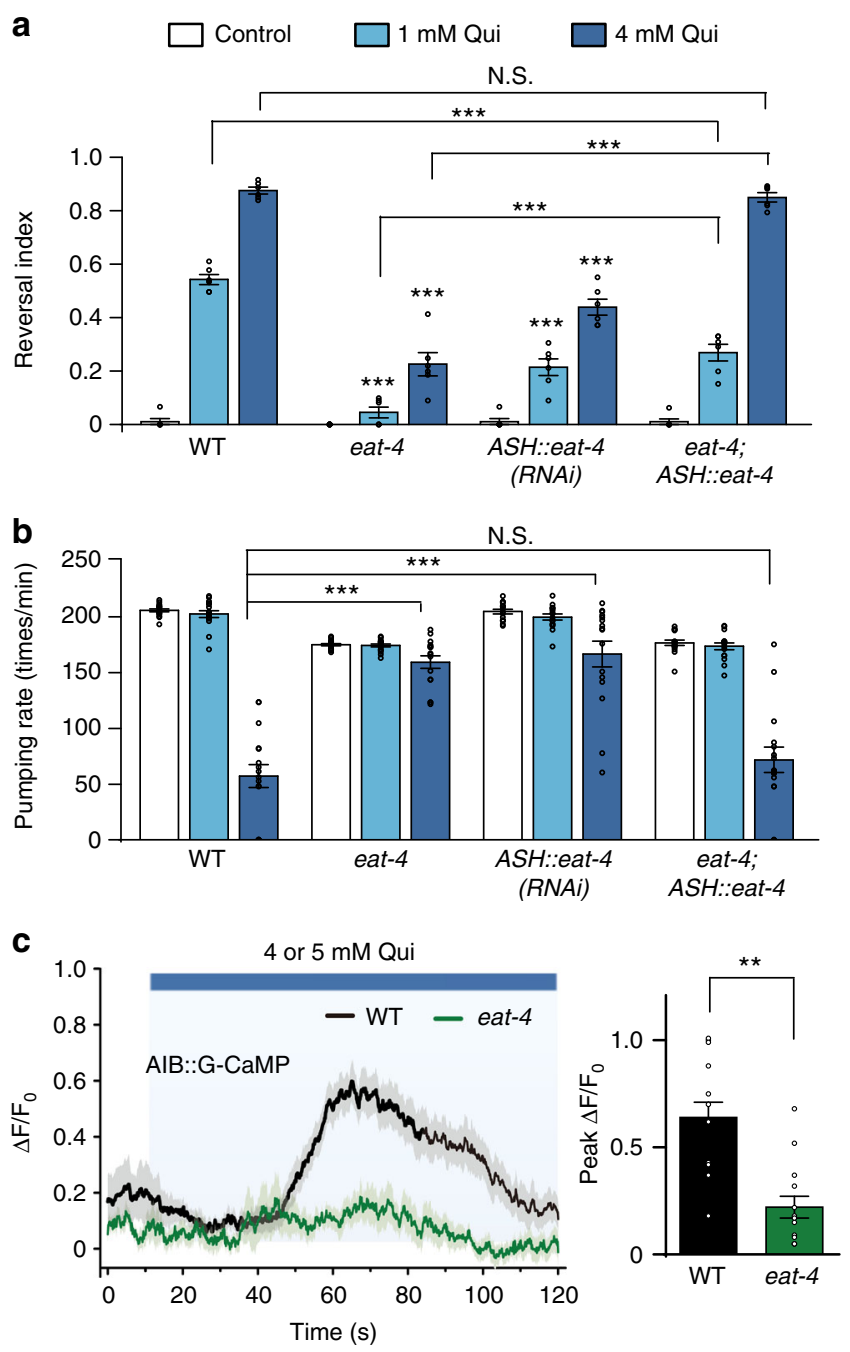

Fig. 3 Glutamate signaling from ASH to AIB mediates both quinine-induced reversal initiation and feeding suppression. $\mathbf{a}$, $\mathbf{b}$ Both reversal initiation and feeding suppression induced by quinine were abolished in eat- 4 mutant worms, and were dramatically declined in worms expressing eat-4 RNAi as a transgene in ASH. Specific expression of wild-type eat-4 gene in ASH partially rescued low-concentration quinine-induced reversal initiation, and fully restored high-concentration quinine-induced reversal initiation (a) and feeding suppression (b). Error bars: s.e.m. Reversal index, $n=6$ groups, 10 worms/group at least; Pumping rate, $n=16$ worms. ${ }^{\star \star \star} p<0.001$ ( $t$ test or Wilcoxon test). c Calcium imaging shown that eat- 4 is required for quinineinduced calcium elevation in AIB. Left: average trace. The shades around traces indicate error bars (s.e.m.). $n \geq 14 .{ }^{\star \star} p<0.01$ ( $t$ test)

required for quinine-induced $\mathrm{Ca}^{2+}$ signaling in AIB. Together, these results suggest that GLR-1 and GLR-5 mediate different intensities of ASH sensory inputs on AIB and two patterns of $\left[\mathrm{Ca}^{2+}\right]_{\mathrm{i}}$ signals, transient and sustained, are differentially regulated by GLR-1 and GLR-5, respectively.

Glutamate-evoked GLR-1 and GLR-5 currents in AIB neurons. Our above behavioral and calcium imaging experiments suggest that GLR-1 and GLR-5 act in AIB to trigger differential behaviors in response to different concentrations of quinine stimulation. Notably, both $g l r-1$ and $g l r-5$ encode putative orthologs of vertebrate non-NMDA glutamate receptors ${ }^{26,27}$. To understand how these two glutamate receptors decode the intensity of quinine inputs, we directly recorded glutamate-evoked electrical responses in the AIB neurons of dissected animals ${ }^{19,26,28-30}$. Since GLR-1, 
a

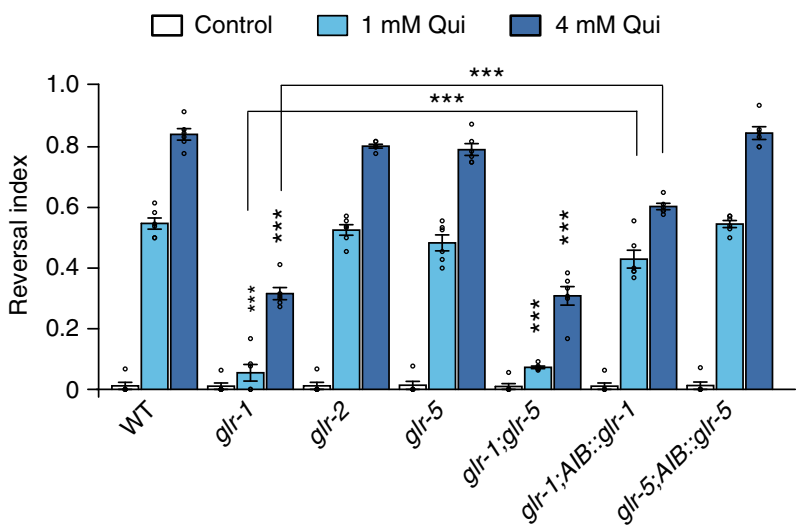

b

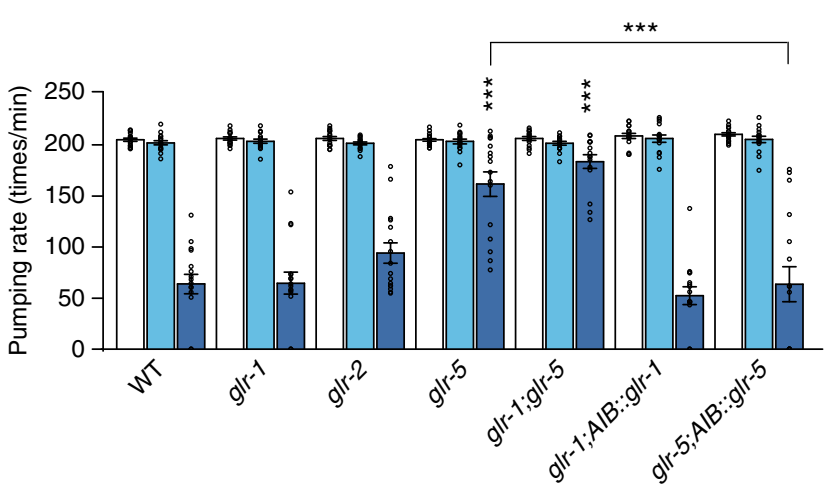

C

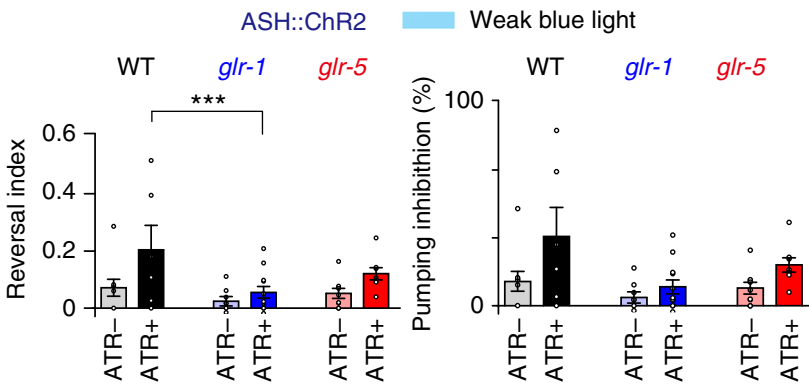

e
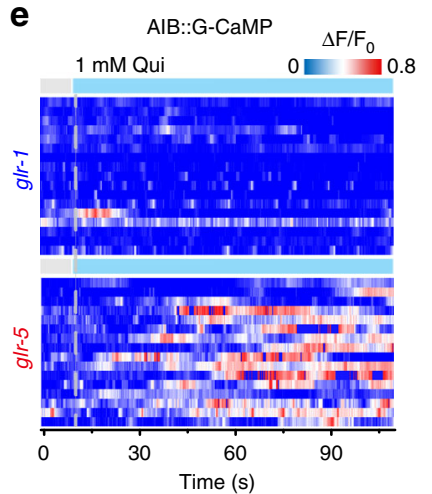

f

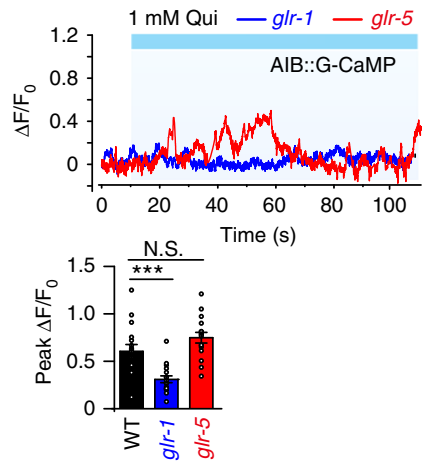

d

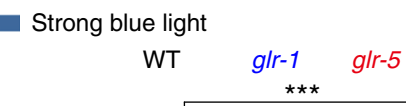

Strong blue light
\[ \text { WT } g / r-1 \quad g / r-5 \]
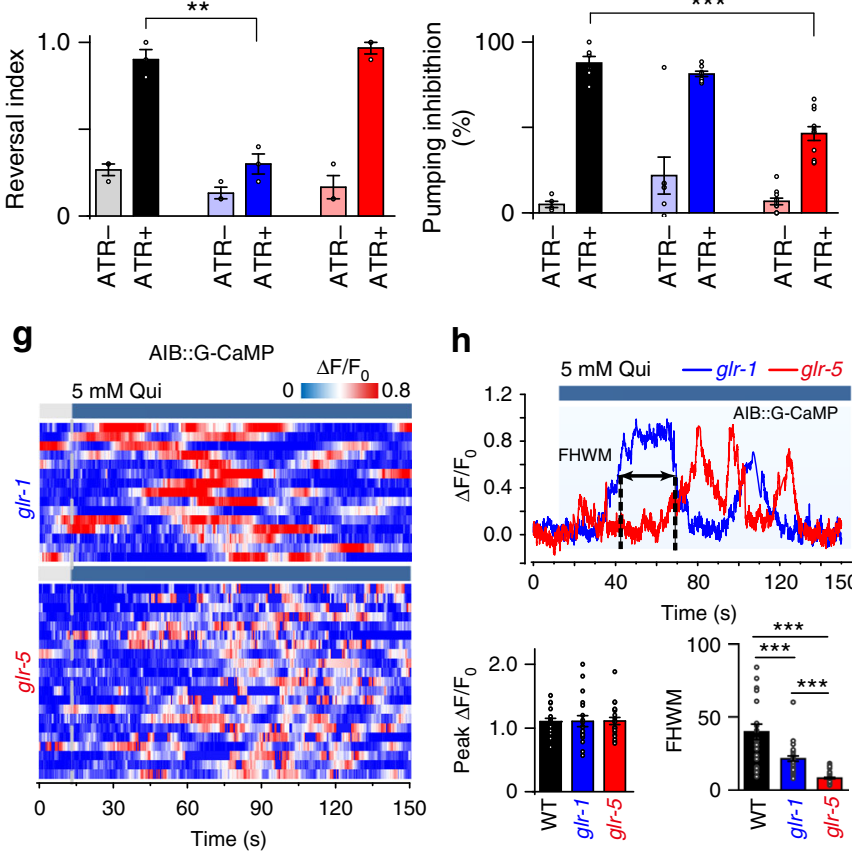

h

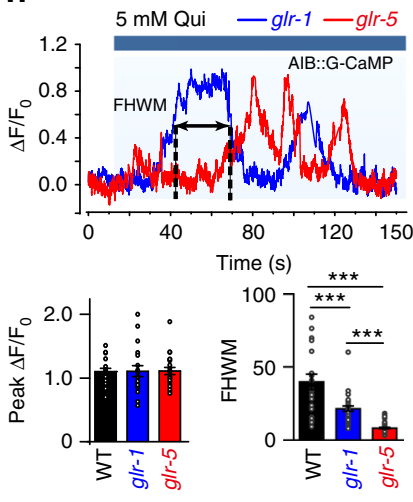

Fig. 4 GLR-1 and GLR-5 in AIB are required for quinine-induced reversal initiation and pumping inhibition, respectively. a glr-1 is required for quinineinduced reversal initiation, which can be rescued by transgenic expression of wild-type g/r-1 gene in AlB. Error bars: s.e.m. $n=6$ groups, 10 worms/group. ${ }^{\star * \star} p<0.001$ ( $t$ test). $\mathbf{b} g / r-5$ is required for quinine-induced pumping inhibition, which can be rescued by transgenic expression of wild-type $g / r-5$ gene in AIB. Error bars: s.e.m. $n=16$ worms. ${ }^{\star \star \star} p<0.001$ ( $t$ test or Wilcoxon test). c Optogenetic stimulation of ASH by blue light $\left(470 \mathrm{~nm} ; 0.03 \mathrm{~mW} \mathrm{~mm}{ }^{-2} ; 30 \mathrm{~s}\right.$ pulse) triggered reversals in wild-type worms, but not in glr-1 mutant worms. The transgenic animals cultured on ATR-free plates were taken as control. d Optogenetic stimulation of ASH by blue light inhibited pumping rate in wild-type worms, but not in glr-5 mutant worms. The transgenic animals cultured on ATR-free plates were taken as control. c, d Error bars: s.e.m.. Reversal index, $n \geq 3$ groups, 10 worms/group; Pumping rate, $n \geq 5$ worms. ${ }^{\star \star \star} p<0.001$ ( $t$ - test). e, $\mathbf{g}$ AIB calcium dynamics induced by low $(\mathbf{e})$ and high concentration of quinine $(\mathbf{g})$, respectively, in freely behaving worms acquired by the iCaN system. Traces are ordered according to the time of quinine application (dash lines). e, $\mathbf{f}$ AIB calcium transients induced by $1 \mathrm{mM}$ quinine were largely abolished in g/r-1 mutant worms. e Heat maps. f Representative traces (upper) and peak calcium changes (lower). Error bars: s.e.m.. $n \geq 16 .{ }^{\star \star \star} p<0.001$ ( $t$ - test). N.S. represents no significant difference. $\mathbf{g}$, h AIB calcium transients induced by $5 \mathrm{mM}$ quinine in glr-1 and g/r-5 mutant worms. $\mathbf{g}$ Heat maps.

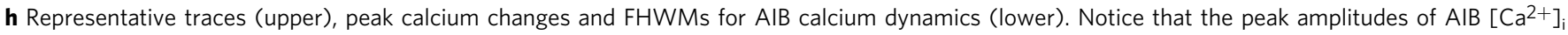
elevations in neither $g / r-1$ nor glr-5 mutant worms shown significant difference from wild-type worms, but the full width at half maximum (FWHM) were much longer in $g / r-1$ mutant worms than that in glr-5. Error bars: s.e.m. $n \geq 17 .{ }^{\star \star} p<0.01 .{ }^{\star \star \star} p<0.001$ (Wilcoxon test)

GLR-5, and AVR-14 glutamate receptors all act in AIB, we set to isolate GLR-1-mediated and GLR-5-mediated current in avr-14; glr-5 and avr-14;glr-1 double mutant background, respectively. To largely retain the intracellular contents, we recorded glutamateinduced currents in the AIB neurons using perforated patchclamp recording. Interestingly, while low concentrations of glutamate induced a rapid activating and fast inactivating inward current in avr-14;glr-5 double mutant (mediated by GLR-1), high concentrations of glutamate evoked a slow activating and more sustained inward current in avr-14;glr-1 double mutant (mediated by GLR-5) (Fig. 5a, b). To further compare the glutamate sensitivity of GLR-1 and GLR-5, we plotted the normalized peak current amplitudes versus glutamate concentrations. As shown in Fig. 5c, glutamate-induced currents in avr-14;glr-5 double mutant background were left-shifted compared to the ones obtained in avr-14;glr-1 double mutant, implying that GLR-1 and GLR-5 have 
a
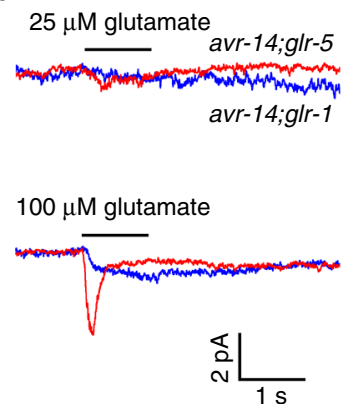
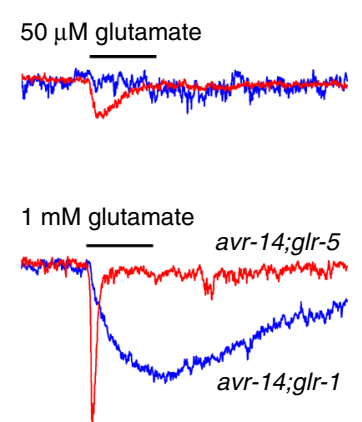
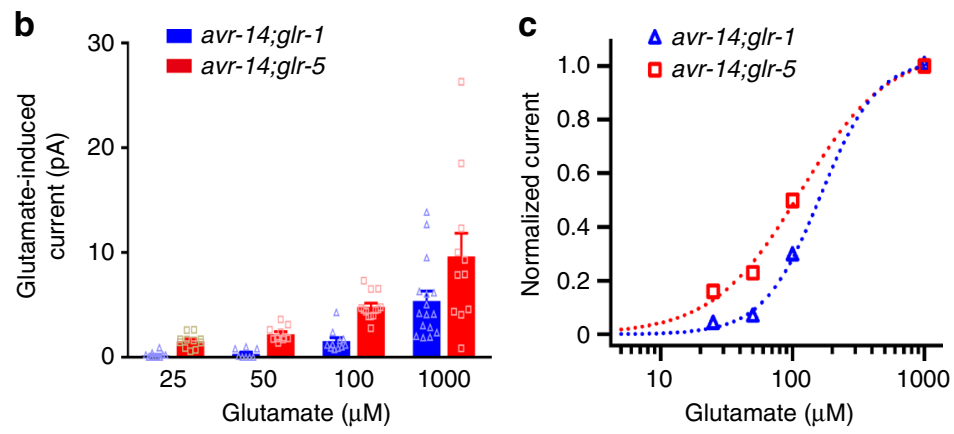

Fig. 5 GLR-1 and GLR-5 currents gated by glutamate have different activation thresholds and kinetics. a Average traces of glutamate-evoked currents recorded in the AIB neurons of $a v r-14 ; g / r-1$ and $a v r-14 ; g / r-5$ double mutant background using perforated patch-clamp recording. A series of concentrations of glutamate $(25 \mu \mathrm{M}-1 \mathrm{mM})$ were perfused to the soma of AIB. AIB neurons were voltage clamped at $-70 \mathrm{mV}$. $n \geq 5$. $\mathbf{b}$ Quantification of the glutamateinduced currents in the AIB neurons of avr-14;glr-1 and avr-14;glr-5 double mutants, respectively. Error bars: s.e.m. $n \geq 5$. c The normalized glutamateinduced currents in the AIB neurons of avr-14;g/r-1 and avr-14; glr-5 double mutants were plotted as a function of the concentrations of glutamate. The tested glutamate concentrations were (in $\mu \mathrm{M}): 25,50,100$, and 1000 . Data were fit with the Hill equation: $I / I$ max $=1 /\left[1+\left(E C_{50} /[g l u t a m a t e]\right)^{n}\right]$, where $n$ represents Hill slope (Hill coefficient)

different sensitivities to glutamate. Notably, the reversal potentials of glutamate-induced currents in the AIB neurons of avr-14;glr-5 and avr-14;glr-1 double mutants were close to $0 \mathrm{mV}$, suggesting that both GLR-1 and GLR-5 are non-selective cation channels (Supplementary Figure 5). Furthermore, kainate $(500 \mu \mathrm{M})$, but not AMPA, evoked similar currents in AIB to the ones induced by glutamate (Supplementary Figure 6), consistent with a previous observation in the AVA neuron ${ }^{30}$. Using a fosmid containing the full length segment of $g l r-5$ genomic DNA linked with GFP, we verified the expression of GLR-5 in AIB (Supplementary Figure 7). Taken together, our above results suggest a thresholdbased mechanism in transmitting different intensities of sensory stimuli from ASH to AIB. In this model, as the quinine concentration increases it causes a gradual activation of ASH and thus a gradual increase in glutamate release. Post-synaptically, different levels of glutamate release are sensed by two glutamate receptors. Lower glutamate levels (i.e., that induced by $1 \mathrm{mM}$ quinine) are sensed by the low-activation threshold GLR-1 receptor, while higher glutamate levels (i.e., that induced by $4-5$ $\mathrm{mM}$ quinine) are sensed by the high-activation threshold GLR-5 receptor. The activation of these two glutamate receptors then triggers reversals and feeding inhibition, respectively.

GLR-5 signals to $\mathrm{Ca}^{2+}$ stores. Since the sensory inputs of quinine bifurcate in AIB through GLR-1 and GLR-5, we were curious as to how these two receptors drive two behavioral outputs from the same neuron. The presence of different $\left[\mathrm{Ca}^{2+}\right]_{i}$ patterns in AIB (Fig. 4e-h) suggests that GLR-1 and GLR-5 activation may trigger $\mathrm{Ca}^{2+}$ signals from different sources. Namely, the GLR-1induced $\left[\mathrm{Ca}^{2+}\right]_{\mathrm{i}}$ elevation is likely mediated, at least in part, by influx through the ionotropic GLR-1 channels ${ }^{16,23}$ themselves. In contrast, large, sustained $\left[\mathrm{Ca}^{2+}\right]_{i}$ elevations are reminiscent of $\mathrm{Ca}^{2+}$ release from $\mathrm{Ca}^{2+}$ stores. We thus examined the potential involvement of intracellular $\mathrm{Ca}^{2+}$ stores in AIB glutamate signaling. We first employed a sarco-endoplasmic reticulum $\mathrm{Ca}^{2+}$-ATPase inhibitor (Thapsigargin, TG) which depletes the intracellular $\mathrm{Ca}^{2+}$ stores $^{31}$. Upon TG $(50 \mu \mathrm{M})$ treatment, the locomotion speed and pharyngeal pumping rate of worms were transiently inhibited and then recovered within $2 \mathrm{~min}$. When we pre-treated glr-1 mutant worms with TG, the highconcentration quinine-induced sustained $\left[\mathrm{Ca}^{2+}\right]_{i}$ elevation in AIB was dramatically reduced, suggesting that the TG-sensitive $\mathrm{Ca}^{2+}$ stores are the major $\mathrm{Ca}^{2+}$ source for GLR-5-mediated $\mathrm{Ca}^{2+}$ signaling. By contrast, quinine-induced $\mathrm{Ca}^{2+}$ transients were unaffected by TG in glr-5 mutant (Fig. 6a), excluding the involvement of TG-sensitive $\mathrm{Ca}^{2+}$ stores in the GLR-1-mediated $\mathrm{Ca}^{2+}$ signaling. Collectively, these results suggest that the sustained $\left[\mathrm{Ca}^{2+}\right]_{\mathrm{i}}$ elevations induced by high concentrations of quinine are largely due to $\mathrm{Ca}^{2+}$ release from $\mathrm{Ca}^{2+}$ stores downstream of GLR-5 activation.

Release of $\mathrm{Ca}^{2+}$ from intracellular stores may be controlled by $\mathrm{IP}_{3}$-mediated signaling acting through the $\mathrm{IP}_{3}$ receptor. In the absence of itr-1 gene which encodes the C. elegans $\mathrm{IP}_{3}$ receptor, sustained $\left[\mathrm{Ca}^{2+}\right]_{i}$ elevations in worms exposed to highconcentration quinine were diminished and became TG-insensitive, short $\left[\mathrm{Ca}^{2+}\right]_{\mathrm{i}}$ transients (Fig. 6a-c), suggesting that ITR-1 is required for high-concentration quinine-induced sustained $\left[\mathrm{Ca}^{2+}\right]_{i}$ response. Consistent with this idea, quinine-induced feeding suppression, but not reversal, was also abolished in itr-1 mutant (Fig. 6d, e). $\mathrm{IP}_{3}$ is produced by members of the phospholipase C (PLC) family. We found that quinine-induced feeding suppression was defective in mutants of two PLC genes, egl ${ }^{-} 8$ (PLC $\left.\beta\right)$ and plc-3 (PLC $\left.\gamma\right)^{32}$. Our above data implied similarities between the reversal duration and feeding suppression. Indeed, we verified that the reversal duration triggered by high concentrations of quinine was mainly impacted by $g l r-5$ and itr-1 mutations, but not by $g l r-1$ mutation, suggesting that both the reversal duration and the pumping rate are controlled mainly by GLR-5 and intracellular $\mathrm{Ca}^{2+}$ stores (Supplementary Figure 8 ).

How does the interneuron AIB signal to its downstream neurons? unc-31 encodes the ortholog of the mammalian CAPS protein, which has been shown to be specifically required for peptide release from dense core vesicles (DCVs) ${ }^{33,34}$. Interestingly, both the loss-of-function mutant of $u n c-31$ and AIBspecific unc-31 RNAi abolished quinine-induced feeding suppression, which can be rescued by the expression of UNC-31 in AIB (Fig. 6d, e). To further confirm the release of DCVs from AIB, we optogenetically stimulated AIB by ChR2. As a control experiment, strong optogenetic activation of AIB elicited feeding suppression in wild-type, eat- 4 and $g l r-1$ mutants, indicating that EAT-4 and GLR-1 are not required for the AIB-mediated feeding suppression. In contrast, strong activation of AIB failed to suppress the pumping rate in either $u n c-31$ or glr-1;unc-31 double mutants (Fig. 6f, g), supporting that the UNC-31-mediated DCVs release is required for the AIB-mediated feeding suppression. Taken together, these data strongly suggest the participation of $\mathrm{Ca}^{2+}$ store-regulated neuropeptide release from DCVs in GLR-5mediated feeding suppression. Notably, our results that GLR-5 signaling is linked to $\mathrm{Ca}^{2+}$ stores are consistent with non-canonic 
a

Control $\quad 50 \mu \mathrm{M}$ TG preincubation $\quad 5 \mathrm{mM}$ Qui
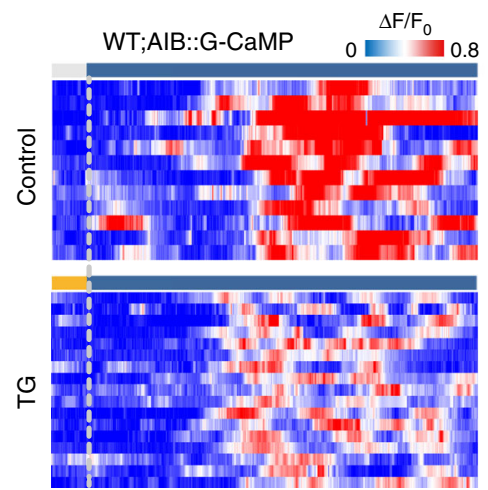

glr-5;AIB::G-CaMP

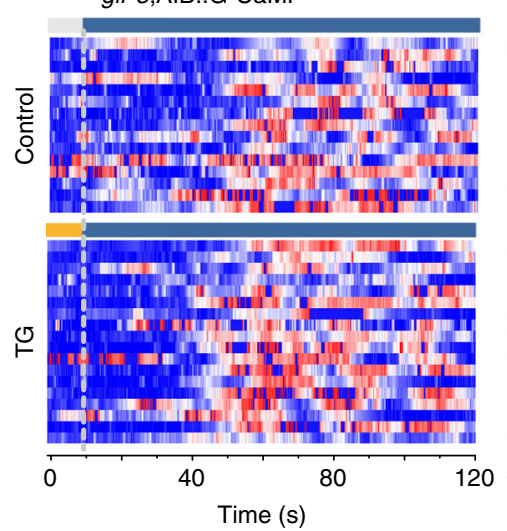

d

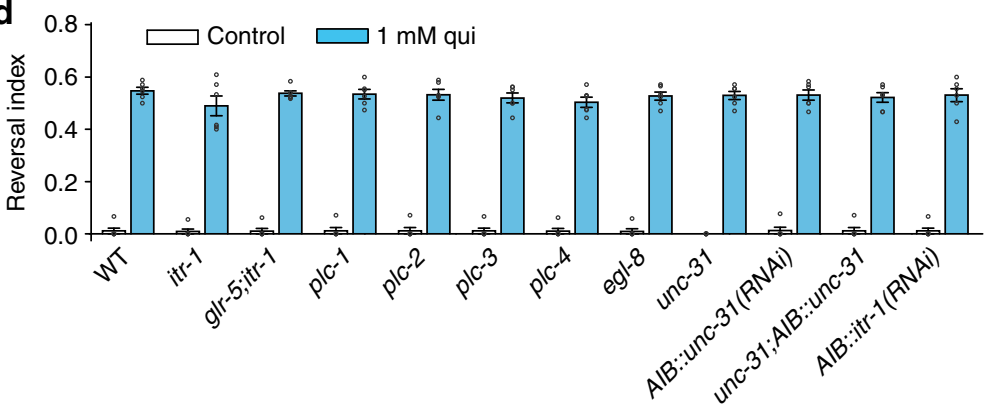

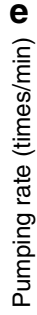

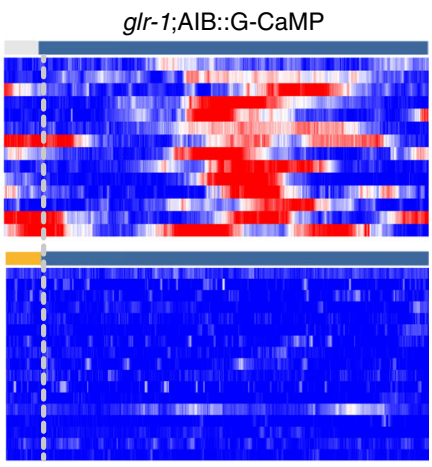

itr-1;AIB::G-CaMP
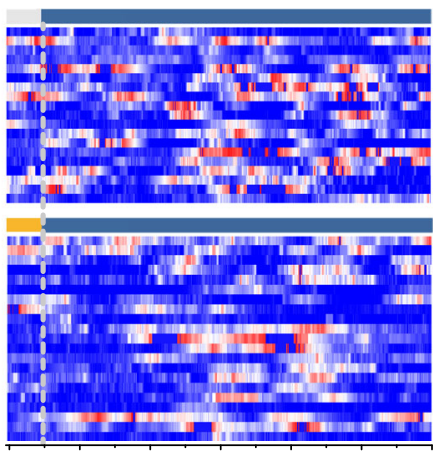

40

80

120

g

AIB::ChR2 Strong blue light

$\square$ Before light $\square$ Light on

glr-1 glr-1;unc-31

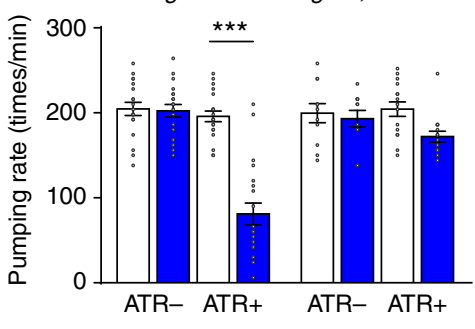

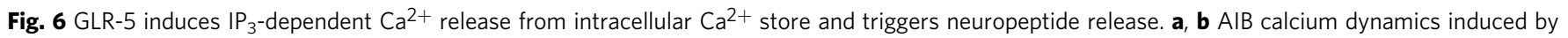

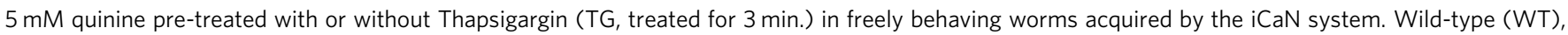

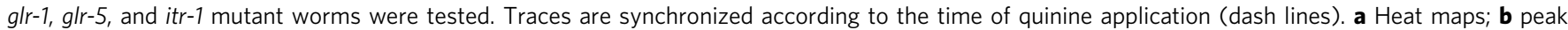
calcium changes, and c FHWMs for AIB calcium dynamics. Error bars: s.e.m.. $n \geq 14 .{ }^{\star \star \star} p<0.001$ ( $t$ test). N.S. represents no significant difference.

d, e Quinine-induced feeding suppression (d), but not reversals (e), was dramatically defective in egl-8, plc-3, itr-1, and unc-31 mutant worms. Error bars: s.e. m.. Reversal index, $n=6$ groups, 10 worms/group at least; Pumping rate, $n=16$ worms. ${ }^{\star \star \star} p<0.001$ ( $t$ test). e Optogenetic stimulation of AlB inhibited

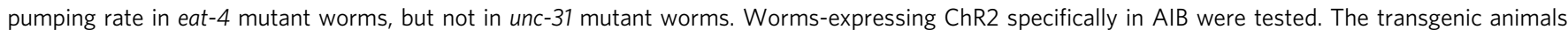
cultured on ATR-free plates were taken as control. Error bars: s.e.m. $n \geq 12 .{ }^{\star \star \star} p<0.001$ (Wilcoxon test). $\mathbf{g}$ Optogenetic stimulation of AIB inhibited pumping rate in glr-1 mutant worms, but not in glr-1;unc-31 mutant worms. Worms expressing ChR2 specifically in AIB were tested. The transgenic animals cultured on ATR-free plates were taken as control. Error bars: s.e.m. $n \geq 12$. ${ }^{\star \star \star} p<0.001$ (Wilcoxon test) 
coupling between vertebrate GIRK-1/GLUR5 and $\mathrm{IP}_{3}$-sensitive $\mathrm{Ca}^{2+}$ stores $^{35}$.

AIB suppresses feeding by activating RIM. The C. elegans wiring diagram predicts bidirectional chemical synapses as well as gap junctions between $\mathrm{AIB}$ and $\mathrm{RIM}^{4,8}$. Previous studies have suggested an important role for RIM in reversals ${ }^{13,15,20,22}$. Here, we further investigated the connection between AIB and RIM during feeding suppression by monitoring $\mathrm{Ca}^{2+}$ levels in RIM. At low concentrations of quinine, we observed little activity in RIM. However, when challenged with high concentrations of quinine, we observed robust $\left[\mathrm{Ca}^{2+}\right]_{i}$ elevations in RIM (Fig. 7a). Since ASH directly synapses to AVA and AVA is also electrically coupled with $\mathrm{RIM}^{15}$, it is possible that the signal from high concentrations of quinine could bypass AIB to activate RIM. To further confirm whether RIM acts downstream of AIB in feeding suppression, we carried out a number of experiments and obtained the following results: (1) Quinine-induced RIM activities were largely abolished in $u n c-31$ mutant (Fig. 7a, b), suggesting an essential role of neuropeptide release in the activation of RIM. (2) Strong activation of AIB by ChR2 elicited robust $\left[\mathrm{Ca}^{2+}\right]_{\mathrm{i}}$ elevations in RIM (Fig. 7c). (3) Direct activation of RIM by ChR2 inhibited pumping rate (Fig. $7 \mathrm{~d}$ ) even in the absence of AIB neurons (Fig. 7e). (4) In contrast to wild-type (Fig. 2e) and eat-4 mutant (Fig. 6e), optogenetic activation of AIB in $t d c-1$ mutant failed to inhibit pumping rate (Fig. 7f). Since $t d c-1$ encodes a tyrosine decarboxylase required to convert tyrosine into tyramine in $\mathrm{RIM}^{8,36}$, AIB may act through tyramine to mediate feeding a

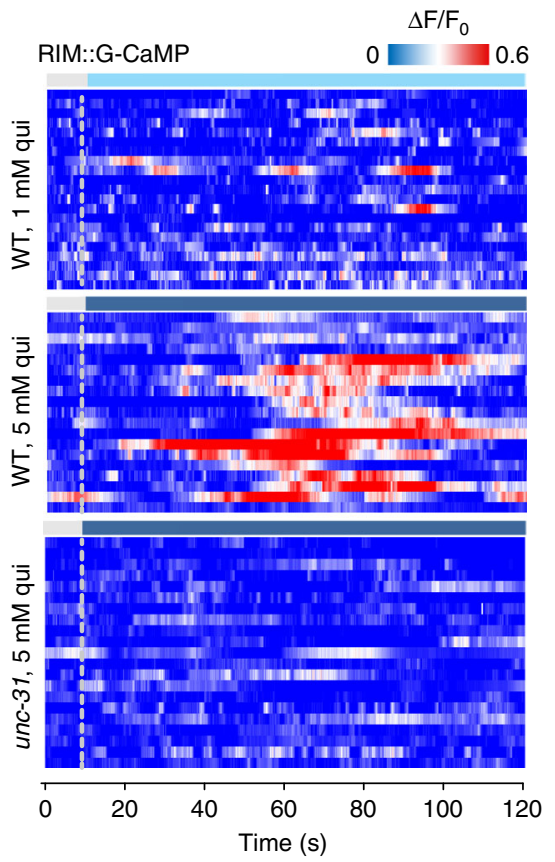

b

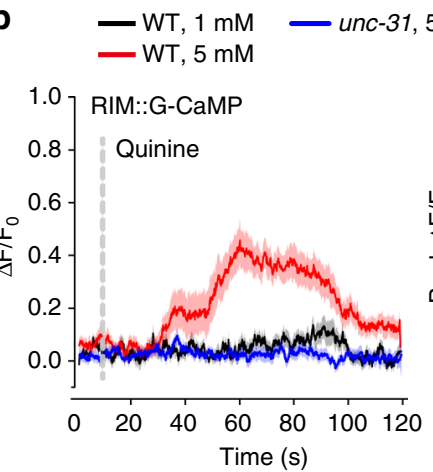

C
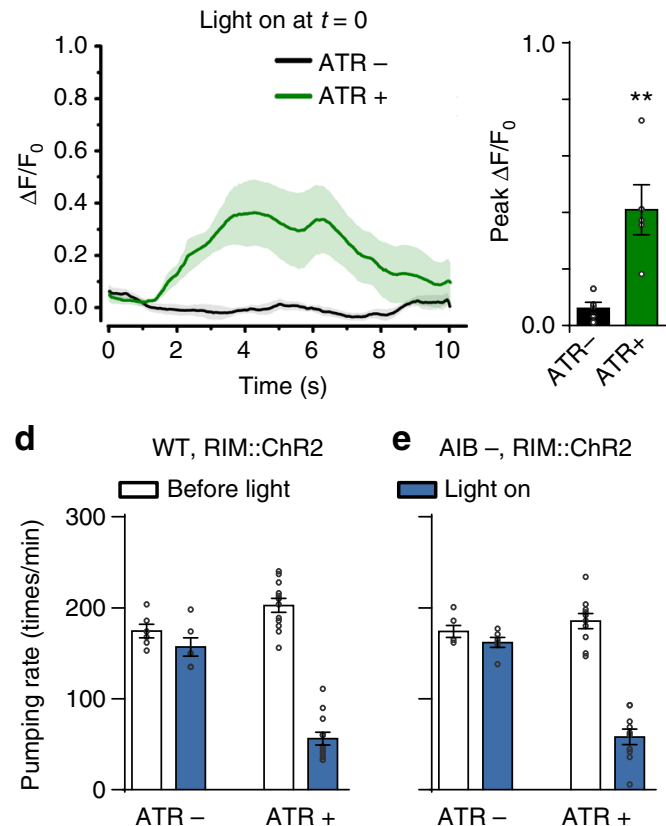

f

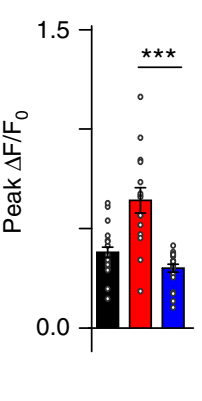

e $\mathrm{AIB}-$, RIM::ChR2
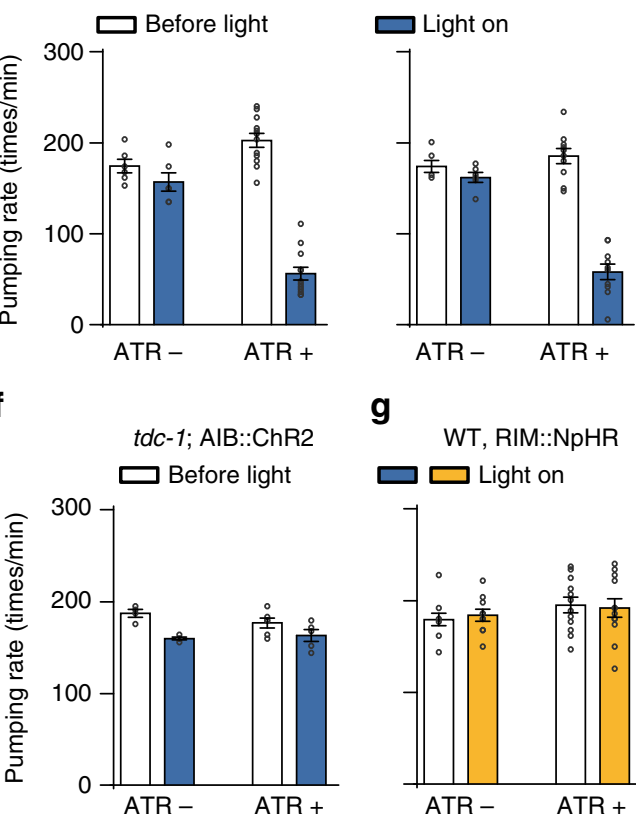

g

WT, RIM::NpHR
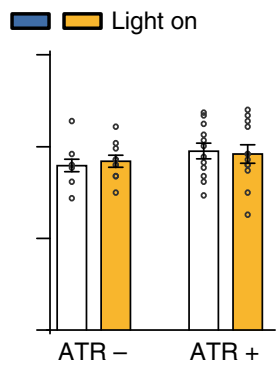

Fig. 7 AIB suppresses feeding by activation of RIM via neuropeptides. $\mathbf{a}$, b RIM calcium dynamics induced by quinine in freely behaving worms acquired by the iCaN system. Traces are synchronized according to the time of quinine application (dash lines). High-concentration quinine promoted the activity of RIM. Absence of unc-31 abolished quinine-induced RIM activities. a Heat maps; b Average traces (left) and peak changes (right) for RIM calcium dynamics. The shades around traces indicate error bars (s.e.m.). $n \geq 15 .{ }^{\star \star \star} p<0.001$ ( $t$ test). c Optogenetic stimulation of AIB promoted the activity of RIM. Worms expressing two transgenes (ChR2 in AIB, and GCaMP in RIM) were tested. The transgenic animals cultured on ATR-free plates were taken as control. Average traces (left) and peak changes (right) for RIM calcium dynamics were shown. The shades around traces indicate error bars (s.e.m.). $n \geq 5$. ${ }^{\star \star} p<$ 0.01 ( $t$ test). d, e Optogenetic stimulation of RIM inhibited pumping rate in both wild-type and AlB-ablated worms. Worms expressing ChR2 specifically in RIM were tested. The transgenic animals cultured on ATR-free plates were taken as control. Error bars: s.e.m. $n \geq 6$. ${ }^{\star \star \star} p<0.001$ ( $t$ test). $\mathbf{f} t d c-1$ is required for RIM mediated pumping inhibition. tdc- 1 mutant worms expressing ChR2 specifically in RIM were tested. The transgenic animals cultured on ATR-free plates were taken as control. Error bars: s.e.m. $n \geq 4$. N.S. represents no significant difference. $\mathbf{g}$ Optogenetic inhibition of RIM produced no effect on pumping. Worms expressing NpHR specifically in RIM were tested. The transgenic animals cultured on ATR-free plates were taken as control. Error bars: s.e.m. $n \geq 10$. N.S. represents no significant difference 
suppression. (5) Inhibition of RIM by a light-gated chloride pump halorhodopsin (NpHR) had no effect on pumping (Fig. 7g). Taken together, these results support that the activation of RIM by AIB is not only sufficient, but also essential in mediating feeding suppression.

\section{Discussion}

Two opposing models have been proposed to explain how neural circuits decode stimulus intensity. According to the pattern theory, a stimulus of sufficient intensity elicits a distinct pattern across functionally indistinct sensory neurons; the resulting pattern is then decoded within the central nervous system to generate a specific behavior output, i.e., avoidance of noxious stimuli $^{37}$. In contrast, the specificity theory posits that specific subtypes of sensory neuron are tuned to detect stimulus intensity via distinct receptors ${ }^{38-40}$. As such, information about the stimulus intensity is encoded, at least in part, by the primary sensory neuron itself, even before signals reach the interneurons. There are a number of examples which illustrate the specificity theory in action. For example, the sodium-selective ENaC channel acts as taste receptor for low-concentration salt and is required for behavioral attraction ${ }^{41,42}$, whereas highconcentration salt induces aversive behaviors which may involve two primary aversive taste pathways by activating the sour and bitter taste-sensing cells ${ }^{1}$. In this study, we have demonstrated a model where the stimulus intensity of quinine was encoded by differential signals flowing in the same neuronal connection. We interrogated the circuitry, synaptic and intracellular mechanisms underlying the decoding of quinine inputs by applying a multifaceted approach integrating $\mathrm{Ca}^{2+}$ imaging, optogenetics and behavioral analysis in freely moving animals together with genetic manipulation, laser and chemical ablation, and electrophysiology.

Our previous work revealed an ASH-RIM axis in the sensation of high-concentration quinine and feeding suppression ${ }^{12}$. However the synaptic connection between ASH and RIM remained to be explored. Here we conclude, based on four lines of evidence that AIB is the interneuron downstream of ASH in mediating feeding inhibition. First, $\left[\mathrm{Ca}^{2+}\right]_{\mathrm{i}}$ activities in AIB correlated with feeding inhibition (Fig.1). Second, ablation of AIB blocked quinine-induced feeding inhibition (Fig. 2). Third, optogenetic activation of AIB inhibited feeding (Fig. 2). Fourth, the identification of the presynaptic neurotransmitter glutamate from ASH and the post-synaptic receptor (GLR-5) on AIB to mediate feeding inhibition. Taken together, our results proposed a previously unidentified role for AIB in feeding regulation.

Interestingly, AIB is also known for its involvement in reversal initiation $15,16,21$. In this study, we confirmed that AIB is required for quinine-induced reversal initiation (Supplementary Figure 2). We showed that low concentration of quinine mediated AIBdependent reversal, whereas high concentration of quinineinduced AIB-dependent reversal and feeding inhibition. How might changes in the intensity of sensory inputs to ASH result in additional behavior outputs through the same AIB interneuron? One interesting feature of C. elegans neurons is that they are nonspiking and activated in a graded manner ${ }^{43}$. Different strength of stimulation of ASH is therefore likely to result in graded glutamate release, which could be decoded in AIB via receptors with different activation threshold and different kinetics for glutamate. Indeed, using single-neuron electrophysiology, we proved that GLR-1 has a lower activation threshold and fast kinetics for glutamate, while GLR-5 has a higher activation threshold and more sustained kinetics (Fig. 5). Hence, a single-analog sensory input generates graded response at $\mathrm{ASH}$, which is then bifurcated at the AIB interneuron via differential responses of GLR-1 and GLR-5 receptors.

GLR-1 belongs to the non-NMDA-type ionotropic glutamate receptor family which can be activated by either glutamate or kainate, and regulates a broad range of physiological functions $15,23,26,28$. It has been suggested that GLR-1 mediates rapid activating and inactivating currents and that $\mathrm{Ca}^{2+}$ permeation through GLR-1 causes an increase in $\left[\mathrm{Ca}^{2+}\right]_{\mathrm{i}}$ of post-synaptic targets $^{23,28}$. In contrast, neither the biophysical and pharmacological properties nor the physiological function of GLR-5 has been reported before. Mammalian GluK1/GluR5 is a kainate-type ionotropic glutamate receptor subunit, which is an important mediator of the pre- and post-synaptic actions of glutamate and has been linked to a number of brain disorders such as epilepsy, schizophrenia, and autism ${ }^{29,44}$. In addition to their channelmediated effects, kainate receptors can signal through an unconventional metabotropic mechanism involving G-proteins and second messengers ${ }^{44}$. Interestingly, it has been demonstrated that kainate can induce a $G$ protein-dependent rise in $\left[\mathrm{Ca}^{2+}\right]_{\mathrm{i}}$ via the $\mathrm{Ca}^{2+}$ release from $\mathrm{IP}_{3}$-sensitive $\mathrm{Ca}^{2+}$ stores ${ }^{35,44,45}$ and can act through $\mathrm{PLC}^{32,44}$. In this study, we demonstrated for the first time a GLR-5-dependent excitatory response in AIB (Fig. 5). Similar to GLR-1, GLR-5 can be activated by both glutamate and kainate (Fig. 5, Supplementary Figure 6). Activation of GLR-5 induced a large and long-lasting $\left[\mathrm{Ca}^{2+}\right]_{i}$ increase that requires $\mathrm{Ca}^{2+}$ release from intracellular stores and components of the $\mathrm{IP}_{3-}$ mediated $\mathrm{Ca}^{2+}$ signaling pathway, i.e., $\mathrm{PLC}$ and the $\mathrm{IP}_{3}$-receptor (ITR-1 in C. elegans).

AIB forms unusually dense synaptic connections to RIM with over 30 synapses between the two neurons ${ }^{4}$. The valence and temporal properties of links between AIB and RIM are ambiguous. Previously it has been suggested that AIB-mediated inhibition of RIM activity during reversals ${ }^{13,15}$. Actually the correlation of activities between AIB, RIM, and AVA is complicated due to the complex forward and backward chemical synapses and gap junctions between these neurons, which form a built-in variability generator triggering random reversals ${ }^{20}$. In contrast to the inhibitory transmission between AIB and RIM, we identified an excitatory connection of AIB-RIM in response to high-concentration quinine. How could a single synapse behave as both inhibitory and excitatory? We think this discrepancy can be reconciled by the differential response to different strength of sensory inputs. At low strength of ASH input, i.e., gentle nose touch or low concentration of quinine, AIB might inhibit RIM via the glutamate-gated $\mathrm{Cl}^{-}$channel AVR-14 ${ }^{15}$. Inhibition of RIM then allows activation AVA and triggers reversal via the so-called disinhibitory circuit ${ }^{15}$. At high concentration of quinine, glutamate release from both ASH and AIB can activate AVA in parallel and initiate reversal as previously suggested ${ }^{15}$. On the other hand, high-concentration quinine also triggers the release of neuropeptide-containing DCVs, which are distant from $\mathrm{Ca}^{2+}$ channels and more sensitive to $\mathrm{Ca}^{2+}$ release from intracellular $\mathrm{Ca}^{2+}$ stores $^{46}$. Neuropeptide release from AIB then excites RIM and induces tyramine-dependent feeding inhibition. In Supplementary Figure 9, we summarized the disinhibitory circuit for reversal (dashed line) and the AIB-RIM excitation circuit for feeding regulation. In fact, our previous results also suggest an inhibition of RIM at normal condition, where RIM receives inhibitory inputs from NSM and is largely locked in an inhibitory state in a "flip-flop" circuit ${ }^{12}$. Hence, the activation of RIM requires strong input, i.e., neuropeptide, to overcome the combined inhibition of 5-HT from NSM ${ }^{12}$ and glutamate from $\mathrm{AIB}^{15}$. Activation of RIM at strong sensory input can activate AVA through gap junctions ${ }^{20,22}$ and is thus correlated with longlasting reversals (Fig. 1d, e). 
One of the most fundamental question in neuroscience is how single neurons encode complex behavioral outputs. A recent study has identified an interesting strategy by which one interneuron, AIY, regulates two distinct behavioral outputs: locomotion speed and direction switch ${ }^{47}$. This strategy employs the same neurotransmitter ACh but utilize distinct post-synaptic ACh receptors on different neurons (RIB and AIZ) to command speed and direction switch, which is more akin to specificity theory. In contrast, the current study reveals a different strategy, more like a pattern theory, where distinct signal patterns are employed in the same neurons. At the ASH-AIB synapse, graded presynaptic glutamate signal is decoded and diverges by activating two postsynaptic glutamate receptors in the same AIB neuron. In AIB interneuron, two distinct $\mathrm{Ca}^{2+}$ patterns were employed to induce different neurotransmitter release, glutamate and neuropeptides. At the AIB-RIM synapse, the valence can be either inhibitory or excitatory depending on the neurotransmitter released from AIB (Supplementary Figure 9). This design might have integration advantages since behaviors are encoded in the same neuron by different intracellular signals.

Apparently, the neuron specificity ${ }^{47}$ and signal specificity (this study) strategies complement each other and contribute to the multifunctional properties of neural circuits. There is still much to learn about how the nervous system decodes and encodes different behavioral outputs. Nevertheless, our findings highlight the importance of determining the valence, strength, and temporal properties at the single-neuron level during connectome construction. Furthermore, our analysis of specific avoidance behaviors in C. elegans may help uncover the mechanisms of polymodal signaling such as nociception in more complex organisms since the involvement of neuropeptides in nociception is highly conserved from worms to mammals ${ }^{48,49}$.

\section{Methods}

Correlated $\mathrm{Ca}^{2+}$ imaging, optogenetics and behavioral tracking. We developed a fast tracking system iCaN (Imaging Calcium activities of Nematodes), as described in Supplementary Figure 1, to simultaneously monitor and manipulate neural activities while analyzing locomotion and feeding behaviors in a freely moving worm. Worms were cultured on Nematode growth medium (NGM) plates at $20^{\circ} \mathrm{C}$ with $E$. coli OP50 using standard procedures. Well-fed young adult hermaphrodites were randomly selected and used for all experiments unless otherwise mentioned. A full list of strains used in this study is available in the Supplemental Methods.

Neuronal calcium activity was measured by detecting changes in fluorescence of GCaMP, a genetically encoded calcium indicator. Fluorescence of mKate2 was used as reference in most experiments. Neuronal activities were manipulated optically by illuminating targeted neurons expressing the optogenetic protein $\mathrm{ChR} 2$ or $\mathrm{NpHR}^{15,22}$.

In general a worm was transferred to a small piece of agar spread with a thin layer of OP50 bacteria. The agar pad was prepared with NGM without cholesterol. Briefly, five layers of sticky labels (thickness measured as $0.5 \mathrm{~mm}$ ) were pasted on the edge of a glass slide. A droplet of liquid agar was added on the slide before sandwiching with another glass slide. As a result, the agar layer has a thickness of $0.5 \mathrm{~mm}$ (different thicknesses of agar pad could be prepared by changing the layers of sticky labels). Next, we cut this agar pad into a $10 \times 10 \mathrm{~mm}^{2}$ right before a worm was transferred onto the pad. The worm was then covered carefully with a cover slip and viewed under the microscope with cover slip down. Five microlitre aliquots of quinine stock solution were dropped on the top of the agar mount and allowed to permeate through agar to reach the worm. To calculate the delivered stimulus intensity, the amount of quinine was divided by the volume of agar. It should be noted that the $20-30 \mathrm{~s}$ delay of $\left[\mathrm{Ca}^{2+}\right]_{\mathrm{i}}$ response to quinine stimuli is due to the diffusion of quinine through the thick agar pad $(0.5 \mathrm{~mm})$ because the lag between ASH and AIB response is quite short (Supplementary Figure 10). Indeed, if we reduced the thickness of agar to one page of sticky label $(0.1 \mathrm{~mm})$, we obtained much faster $\left[\mathrm{Ca}^{2+}\right]_{\mathrm{i}}$ responses in ASH and AIB (Supplementary Figure 10). To define $\left[\mathrm{Ca}^{2+}\right]_{\mathrm{i}}$ peaks, the $10 \mathrm{~s}$ baseline response before quinine stimulation was averaged as basal $\left[\mathrm{Ca}^{2+}\right]_{\mathrm{i}}$ and the maximal increase of $\left[\mathrm{Ca}^{2+}\right]_{\mathrm{i}}$ level after quinine stimulation was defined as the $\left[\mathrm{Ca}^{2+}\right]_{\mathrm{i}}$ peak response. If multiple $\left[\mathrm{Ca}^{2+}\right]_{\mathrm{i}}$ peaks are present in one cell, we selected the largest one as the peak response.

For optogenetic experiments, worms were cultured on NGM plates supplied with OP50 and $5 \mathrm{mM}$ all-trans retinal (ATR) and the same transgenic animals cultured on ATR-free plates were used as control. All imaging experiments and optogenetic manipulations were performed on lite-1(xu7) or lite-1(ce314) genetic background to eliminate the intrinsic photophobic response ${ }^{50}$. Illumination was applied by blue light $\sim 488 \mathrm{~nm}$ in $30 \mathrm{~s}$ pulses. For AIB activation, strong blue light was about $1.77 \mathrm{~mW} \mathrm{~mm}^{-2}$ and weak blue light was $0.28 \mathrm{~mW} \mathrm{~mm}^{-2}$. For ASH activation, the light power was $0.2 \mathrm{~mW} \mathrm{~mm}^{-2}$ and $0.03 \mathrm{~mW} \mathrm{~mm}^{-2}$, respectively.

Pumping rate and locomotion were monitored by the iCaN system and assayed using a custom-developed software as described in Supplemental Methods.

Cross-correlation analyses were performed with Origin Lab Pro.9.0.

Laser ablation. Laser ablation was performed on L1 or L2 stage worms by standard procedures $^{51}$. A pulsed laser was used to ablate target neurons. Ablation was confirmed the following day and worms were used for experiments as young adults.

Electrophysiology. Electrophysiological recordings were carried out on an Olympus microscope (BX51WI) with an EPC-10 amplifier and Patchmaster software (HEKA). Day 2 adult worms were glued on the surface of Sylgard-coated coverslips using cyanoacrylate-based glue. A dorsolateral incision was made using sharp glass pipettes to expose the AIB neurons marked by GCaMP3.0 and mKate $2^{29,30,50}$. The bath solution contained (in $\mathrm{mM}$ ) $145 \mathrm{NaCl}, 2.5 \mathrm{KCl}, 5 \mathrm{CaCl}_{2}, 1$ $\mathrm{MgCl}_{2}, 20$ glucose (325-335 mOsm, $\mathrm{pH}$ adjusted to 7.3). The intracellular solution (ICF) contained (in $\mathrm{mM}$ ) $145 \mathrm{~K}$-gluconate, $5 \mathrm{MgCl}_{2}, 5 \mathrm{EGTA}, 0.25 \mathrm{CaCl}_{2}, 10$ HEPES, 10 glucose, $5 \mathrm{Na}_{2} \mathrm{ATP}$, and $0.5 \mathrm{NaGTP}$ (315-325 mOsm, pH adjusted to 7.2). High $\mathrm{Cl}^{-}$ICF: same as ICF with the following changes: $145 \mathrm{mM} \mathrm{KCl}$. For perforated recording, $120 \mu \mathrm{g} \mathrm{mL}^{-1}$ of nystatin was included in ICF. Membrane potential was clamped at $-70 \mathrm{mV}$. Glutamate and kainate were diluted in bath solution and perfused toward the soma of the AIB neuron using a MINJ-D Digital Positive Displacement System (Tritech).

Statistical analysis. Data are expressed as the mean \pm s.e.m., and the statistical significance of differences was assessed using the two-sided $t$ test when the data meet the assumptions of the $t$ test. If the data did not meet the normal distribution, the Wilcoxon test was used. The sample sizes were determined by the reproducibility of the experiments and are similar to those generally employed in the field.

\section{Data availability}

iCaN system used a java-based custom software. Pharyngeal pumping rate (feeding behavior) was quantified using a java-based custom software. The codes are available from the authors upon request. All relevant data are available from the authors upon request.

Received: 20 November 2016 Accepted: 6 September 2018 Published online: 17 October 2018

\section{References}

1. Oka, Y., Butnaru, M., von Buchholtz, L., Ryba, N. J. \& Zuker, C. S. High salt recruits aversive taste pathways. Nature 494, 472-475 (2013).

2. Sasakura, H. \& Mori, I. Behavioral plasticity, learning, and memory in $C$. elegans. Curr. Opin. Neurobiol. 23, 92-99 (2013).

3. Zhang, Y. V., Ni, J. \& Montell, C. The molecular basis for attractive salt-taste coding in Drosophila. Science 340, 1334-1338 (2013).

4. White, J. G., Southgate, E., Thomson, J. N. \& Brenner, S. The structure of the nervous system of the nematode Caenorhabditis elegans. Philos. Trans. R. Soc. Lond. B. Biol. Sci. 314, 1-340 (1986).

5. Bendesky, A. et al. Long-range regulatory polymorphisms affecting a GABA receptor constitute a quantitative trait locus (QTL) for social behavior in Caenorhabditis elegans. PLoS Genet. 8, e1003157 (2012).

6. de Bono, M. \& Maricq, A. V. Neuronal substrates of complex behaviors in $C$ elegans. Annu. Rev. Neurosci. 28, 451-501 (2005).

7. Hobert, O. Behavioral plasticity in C. elegans: paradigms, circuits, genes. $J$. Neurobiol. 54, 203-223 (2003).

8. Jin, X., Pokala, N. \& Bargmann, C. I. Distinct circuits for the formation and retrieval of an imprinted olfactory memory. Cell 164, 632-643 (2016).

9. Neal, S. J. et al. Feeding state-dependent regulation of developmental plasticity via CaMKI and neuroendocrine signaling. eLife 4, e10110 (2015).

10. Li, W., Kang, L., Piggott, B. J., Feng, Z. \& Xu, X. Z. The neural circuits and sensory channels mediating harsh touch sensation in Caenorhabditis elegans. Nat. Commun. 2, 315 (2011).

11. Hilliard, M. A., Bergamasco, C., Arbucci, S., Plasterk, R. H. \& Bazzicalupo, P. Worms taste bitter: ASH neurons, QUI-1, GPA-3 and ODR-3 mediate quinine avoidance in Caenorhabditis elegans. EMBO J. 23, 1101-1111 (2004).

12. Li, Z. et al. Dissecting a central flip-flop circuit that integrates contradictory sensory cues in C. elegans feeding regulation. Nat. Commun. 3, 776 (2012). 
13. Gray, J. M., Hill, J. J. \& Bargmann, C. I. A circuit for navigation in Caenorhabditis elegans. Proc. Natl Acad. Sci. USA 102, 3184-3191 (2005).

14. Hart, A. C., Sims, S. \& Kaplan, J. M. Synaptic code for sensory modalities revealed by C. elegans GLR-1 glutamate receptor. Nature 378, 82-85 (1995).

15. Piggott, B. J., Liu, J., Feng, Z., Wescott, S. A. \& Xu, X. Z. The neural circuits and synaptic mechanisms underlying motor initiation in C. elegans. Cell 147, 922-933 (2011).

16. Summers, P. J. et al. Multiple sensory inputs are extensively integrated to modulate nociception in C. elegans. J. Neurosci. 35, 10331-10342 (2015).

17. Zheng, Y., Brockie, P. J., Mellem, J. E., Madsen, D. M. \& Maricq, A. V. Neuronal control of locomotion in C. elegans is modified by a dominant mutation in the GLR-1 ionotropic glutamate receptor. Neuron 24, 347-361 (1999).

18. Bargmann, C. I. Chemosensation in C. elegans. WormBook. 1-29 https://doi. org/10.1895/wormbook.1.123.1 (2006).

19. Chalfie, M. et al. The neural circuit for touch sensitivity in Caenorhabditis elegans. J. Neurosci. 5, 956-964 (1985).

20. Gordus, A., Pokala, N., Levy, S., Flavell, S. W. \& Bargmann, C. I. Feedback from network states generates variability in a probabilistic olfactory circuit. Cell 161, 215-227 (2015).

21. Luo, L. et al. Dynamic encoding of perception, memory, and movement in a $C$. elegans chemotaxis circuit. Neuron 82, 1115-1128 (2014).

22. Guo, Z. V., Hart, A. C. \& Ramanathan, S. Optical interrogation of neural circuits in Caenorhabditis elegans. Nat. Methods 6, 891-896 (2009).

23. Chalasani, S. H. et al. Dissecting a circuit for olfactory behaviour in Caenorhabditis elegans. Nature 450, 63-70 (2007).

24. Lee, R. Y., Sawin, E. R., Chalfie, M., Horvitz, H. R. \& Avery, L. EAT-4, a homolog of a mammalian sodium-dependent inorganic phosphate cotransporter, is necessary for glutamatergic neurotransmission in Caenorhabditis elegans. J. Neurosci. 19, 159-167 (1999).

25. Brockie, P. J., Madsen, D. M., Zheng, Y., Mellem, J. \& Maricq, A. V. Differential expression of glutamate receptor subunits in the nervous system of Caenorhabditis elegans and their regulation by the homeodomain protein UNC-42. J. Neurosci. 21, 1510-1522 (2001).

26. Brockie, P. J. \& Maricq, A. V. Ionotropic glutamate receptors: genetics, behavior and electrophysiology. WormBook. 1-16, https://doi.org/10.1895/ wormbook.1.61.1 (2006)

27. Shaye, D. D. \& Greenwald, I. OrthoList: a compendium of C. elegans genes with human orthologs. PLoS ONE 6, e20085 (2011).

28. Mellem, J. E., Brockie, P. J., Zheng, Y., Madsen, D. M. \& Maricq, A. V. Decoding of polymodal sensory stimuli by postsynaptic glutamate receptors in C. elegans. Neuron 36, 933-944 (2002)

29. Yang, X. et al. Syntaxin opening by the MUN domain underlies the function of Munc13 in synaptic-vesicle priming. Nat. Struct. Mol. Biol. 22, 547-554 (2015).

30. Yue, X. et al. TMC proteins modulate egg laying and membrane excitability through a background leak conductance in C. elegans. Neuron 97, 571-585 (2018).

31. Singaravelu, G. et al. The sperm surface localization of the TRP-3/SPE-41 Ca2 + -permeable channel depends on SPE-38 function in Caenorhabditis elegans. Dev. Biol. 365, 376-383 (2012).

32. Baylis, H. A. \& Vazquez-Manrique, R. P. Genetic analysis of IP3 and calcium signalling pathways in C. elegans. Biochim. Et. Biophys. Acta 1820, 1253-1268 (2012).

33. Speese, S. et al. UNC-31 (CAPS) is required for dense-core vesicle but not synaptic vesicle exocytosis in Caenorhabditis elegans. J. Neurosci. 27, 6150-6162 (2007).

34. Zhou, K. M. et al. PKA activation bypasses the requirement for UNC-31 in the docking of dense core vesicles from C. elegans neurons. Neuron 56, 657-669 (2007).

35. Ouardouz, M. et al. Glutamate receptors on myelinated spinal cord axons: II. AMPA and GluR5 receptors. Ann. Neurol. 65, 160-166 (2009).

36. Alkema, M. J., Hunter-Ensor, M., Ringstad, N. \& Horvitz, H. R. Tyramine functions independently of octopamine in the Caenorhabditis elegans nervous system. Neuron 46, 247-260 (2005).

37. Melzack, R. \& Wall, P. D. Pain mechanisms: a new theory. Science 150, 971-979 (1965).

38. Bessou, P. \& Perl, E. R. Response of cutaneous sensory units with unmyelinated fibers to noxious stimuli. J. Neurophysiol. 32, 1025-1043 (1969).

39. Julius, D. TRP channels and pain. Annu. Rev. Cell Dev. Biol. 29, 355-384 (2013).

40. Sherrington, C. S. Qualitative difference of spinal reflex corresponding with qualitative difference of cutaneous stimulus. J. Physiol. 30, 39-46 (1903).

41. Chandrashekar, J. et al. The cells and peripheral representation of sodium taste in mice. Nature 464, 297-301 (2010).

42. Liu, L. et al. Contribution of Drosophila DEG/ENaC genes to salt taste. Neuron 39, 133-146 (2003).
43. Liu, P., Chen, B. \& Wang, Z. W. Postsynaptic current bursts instruct action potential firing at a graded synapse. Nat. Commun. 4, 1911 (2013).

44. Lerma, J. \& Marques, J. M. Kainate receptors in health and disease. Neuron 80, 292-311 (2013).

45. Rodriguez-Moreno, A. \& Sihra, T. S. Presynaptic kainate receptor-mediated facilitation of glutamate release involves $\mathrm{Ca} 2+-$ calmodulin and PKA in cerebrocortical synaptosomes. FEBS Lett. 587, 788-792 (2013).

46. Stigloher, C., Zhan, H., Zhen, M., Richmond, J. \& Bessereau, J. L. The presynaptic dense projection of the Caenorhabiditis elegans cholinergic neuromuscular junction localizes synaptic vesicles at the active zone through SYD-2/Liprin and UNC-10/RIM-dependent interactions. J. Neurosci. 31, 4388-4396 (2011).

47. Li, Z., Liu, J., Zheng, M. \& Xu, X. Z. Encoding of both analog- and digital-like behavioral outputs by one C. elegans interneuron. Cell 159, 751-765 (2014).

48. Mills, H., Hapiak, V., Harris, G., Summers, P. \& Komuniecki, R. The interaction of octopamine and neuropeptides to slow aversive responses in $C$. elegans mimics the modulation of chronic pain in mammals. Worm $\mathbf{1}$, 202-206 (2012).

49. Scherrer, G. et al. Dissociation of the opioid receptor mechanisms that control mechanical and heat pain. Cell 137, 1148-1159 (2009).

50. Liu, J. et al. C. elegans phototransduction requires a $G$ protein-dependent cGMP pathway and a taste receptor homolog. Nat. Neurosci. 13, 715-722 (2010).

51. Fang-Yen, C., Gabel, C. V., Samuel, A. D., Bargmann, C. I. \& Avery, L. Laser microsurgery in Caenorhabditis elegans. Methods Cell Biol. 107, 177-206 (2012).

\section{Acknowledgements}

We would like to thank L. Looger, the Caenorhabditis Genetic Center, and Addgene for strains and constructs. We are grateful for critical reading and comments from M.C.

Wang and X.Z. Shawn Xu. This work was supported by the National Key Research and Development Program of China (2016YFA0500200, 2017YFA0505300, 2016YFA0502400), the National Foundation of Natural Science of China (31730054, 31771113, 31271180, 31471023), Zhejiang Provincial Natural Science Funds of China (LR14C090001), the Fundamental Research Funds for the Central Universities of China (2017FZA7003 and 2018FZA7004), and the Non-profit Central Research Institute Fund of Chinese Academy of Medical Sciences (2017PT31038 and 2018РT31041).

\section{Author contributions}

T.X., H.Z. and L.K. designed experiments. H.Z. developed the iCaN system and data analyzing software. H.Z., J.F., K.D., W.H., J.Y., S.L. and Y.F. conducted calcium imaging, optogenetics, and behavioral experiments. W.Z. conducted behavioral and electrophysiological experiments. T.X., H.Z., W.J., R.X., S.G. and L.K. analyzed and interpreted results. T.X., L.K., H.B. and H.Z. wrote the manuscript, which was modified by all other authors.

\section{Additional information}

Supplementary Information accompanies this paper at https://doi.org/10.1038/s41467018-06819-5.

Competing interests: The authors declare no competing interests.

Reprints and permission information is available online at http://npg.nature.com/ reprintsandpermissions/

Publisher's note: Springer Nature remains neutral with regard to jurisdictional claims in published maps and institutional affiliations.

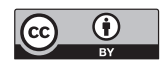

Open Access This article is licensed under a Creative Commons Attribution 4.0 International License, which permits use, sharing, adaptation, distribution and reproduction in any medium or format, as long as you give appropriate credit to the original author(s) and the source, provide a link to the Creative Commons license, and indicate if changes were made. The images or other third party material in this article are included in the article's Creative Commons license, unless indicated otherwise in a credit line to the material. If material is not included in the article's Creative Commons license and your intended use is not permitted by statutory regulation or exceeds the permitted use, you will need to obtain permission directly from the copyright holder. To view a copy of this license, visit http://creativecommons.org/ licenses/by/4.0/.

(C) The Author(s) 2018 